\title{
Algebraic properties of the monopole formula
}

\section{Amihay Hanany ${ }^{a}$ and Marcus Sperling ${ }^{b}$}

a Theoretical Physics Group, Imperial College London,

Prince Consort Road, London, SW7 2AZ, U.K.

${ }^{b}$ Fakultät für Physik, Universität Wien,

Boltzmanngasse 5, 1200 Wien, Austria

E-mail: a.hanany@imperial.ac.uk, marcus.sperling@univie.ac.at

ABSTRACT: The monopole formula provides the Hilbert series of the Coulomb branch for a 3-dimensional $\mathcal{N}=4$ gauge theory. Employing the concept of a fan defined by the matter content, and summing over the corresponding collection of monoids, allows the following: firstly, we provide explicit expressions for the Hilbert series for any gauge group. Secondly, we prove that the order of the pole at $t=1$ and $t \rightarrow \infty$ equals the complex or quaternionic dimension of the moduli space, respectively. Thirdly, we determine all bare and dressed BPS monopole operators that are sufficient to generate the entire chiral ring. As an application, we demonstrate the implementation of our approach to computer algebra programs and the applicability to higher rank gauge theories.

KEywords: Field Theories in Lower Dimensions, Supersymmetric gauge theory

ARXIV EPRINT: 1611.07030 


\section{Contents}

1 Introduction 2

2 Monoids and associated algebras $\quad 4$

2.1 Geometric picture 4

2.1.1 Cones 4

2.1.2 Monoids 5

$\begin{array}{lll}2.2 & \text { Algebraic picture } & 6\end{array}$

2.3 Hilbert series $\quad 7$

2.3.1 Hilbert series for a monoid 8

2.3.2 Hilbert series for the relative interior of a monoid 9

2.4 Free resolution 9

$\begin{array}{lll}2.4 .1 & \text { Free resolution } & 10\end{array}$

$\begin{array}{lll}2.4 .2 & \text { Graded free resolution } & 11\end{array}$

2.5 Canonical module 11

3 Casimir invariance 12

4 Application to monopole formula $\quad \mathbf{1 5}$

$\begin{array}{lll}4.1 & \text { Simplicial refinements } & 17\end{array}$

$\begin{array}{lll}4.2 & \text { Free resolutions } & 17\end{array}$

$\begin{array}{ll}4.3 \text { Canonical module } & 19\end{array}$

4.4 Comments on matter fan 20

4.5 Product gauge groups 21

5 Pole structure of the monopole formula $\quad 22$

$\begin{array}{ll}5.1 \text { Order of pole at } t=1 & 22\end{array}$

5.2 Order of pole at $t \rightarrow+\infty \quad 22$

$\begin{array}{ll}5.3 \text { Order of pole at } t=-1 & 23\end{array}$

5.4 Order of pole at other roots of unity 24

6 Chiral ring generators $\quad 25$

$\begin{array}{lll}7 & \text { Implementation } & \mathbf{2 7}\end{array}$

$\begin{array}{lll}7.1 \text { A recipe } & 27\end{array}$

$\begin{array}{lll}7.2 & \text { Example quiver gauge theories } & 28\end{array}$

$\begin{array}{lll}\text { 7.2.1 } & \text { Example I: rank } 3 & 28\end{array}$

$\begin{array}{lll}7.2 .2 & \text { Example II: rank } 4 & 31\end{array}$

7.2.3 Example III: rank $6 \quad 33$

8 Conclusions $\quad 36$

$\begin{array}{ll}\text { A Reminder: algebraic geometry } & 37\end{array}$ 


\section{Introduction}

The moduli spaces of 3-dimensional supersymmetric gauge theories with 8 supercharges have revealed various interesting features. The two prominent branches, Coulomb $\mathcal{M}_{C}$ and Higgs $\mathcal{M}_{H}$, lie both in the family of hyper-Kähler spaces, but behave fundamentally different as exhibited in their dimension and behaviour under quantum-corrections, for instance.

There have been various attempts to understand the Coulomb branch from a variety of perspectives. Here, we focus on the viewpoint introduced in [1], which introduced the monopole formula as a prescription of the Hilbert series for the chiral ring $\mathbb{C}\left[\mathcal{M}_{C}\right]$. This approach has been applied to various questions [2-6], and found extensions to $\mathcal{N} \geq 2$ theories [7-9] as well as mixed branches [10]. Other approaches attempt a mathematically rigorous definition of the Coulomb branch [11-13] or study the quantised chiral ring $[14,15]$.

Monopole formula. We recall the monopole formula for a 3 -dimensional $\mathcal{N}=4$ gauge theory with gauge group $\mathrm{G}$ as

$$
\mathrm{HS}_{\mathrm{G}}(t)=\sum_{m \in \Lambda_{w}(\widehat{\mathrm{G}}) / \mathcal{W}_{\widehat{\mathrm{G}}}} t^{\Delta(m)} P_{\mathrm{G}}(t ; m),
$$

wherein $\Lambda_{w}(\widehat{\mathrm{G}})$ is the weight lattice of the GNO-dual group $\widehat{\mathrm{G}}$, and $\mathcal{W}_{\widehat{\mathrm{G}}}$ denotes the Weyl group. As shown in [16], the lattice $\Lambda_{w}(\widehat{\mathrm{G}})$ coincides with the solutions of the generalised Dirac quantisation condition [17]. The way $\mathrm{HS}_{\mathrm{G}}$ realises the Hilbert series is by counting BPS monopole operators, as studied by [18-20]. A key ingredient is the existence and uniqueness of the bare BPS monopole operator for each point in $\Lambda_{w}(\widehat{\mathrm{G}})$ [19]. These operators can be further characterised by their conformal dimension $\Delta(m)$, which is given by $[19,21-23]$

$$
\Delta(m)=\frac{1}{2} \sum_{i=1}^{n} \sum_{\rho \in \mathcal{R}_{i}}|\rho(m)|-\sum_{\alpha \in \Phi_{+}}|\alpha(m)| .
$$

Here, $\Phi_{+}$denotes the set of positive roots of $\mathfrak{g}=\operatorname{Lie}(G)$, and $\mathcal{R}_{i}$ is the set of all weights of the G-representation the $i$-th flavour of the $\mathcal{N}=4$ hyper-multiplets transform in. We restrict our attention to good theories in the sense of [21], i.e. all non-trivial BPS monopole operators satisfy $\Delta>\frac{1}{2}$. Lastly, compatibility with $\mathcal{N}=4$ supersymmetry allows for a non-vanishing vacuum expectations value of a complex linear combination of the adjoint values scalar fields in the $\mathcal{N}=4$ vector multiplet. The precise condition is that the vacuum expectation values can be any polynomial on the Lie algebra of the residual gauge group $\mathrm{H}_{m}=\operatorname{Stab}_{\mathrm{G}}(m)$ which has to be invariant under $\mathrm{H}_{m}$. This gives rise to the dressing factors $P_{\mathrm{G}}(t ; m)$ which are understood as Poincaré series of the algebra of $\mathrm{H}_{m}$ invariant polynomials on $\operatorname{Lie}\left(\mathrm{H}_{m}\right)$, see for instance [6, section 2].

The matter fan and Hilbert bases. In [6] we introduced geometric concepts that, on the one hand, may simplify or at least systematise the computations for the monopole formula. On the other hand, the presented approach might lead to a better understand of the Coulomb branch itself. 
The first step is just group and representation theory. The dominant Weyl chamber $\sigma$ of the GNO-dual group $\widehat{\mathrm{G}}$ is a rational polyhedral cone inside a Cartan subalgebra $\mathfrak{t}$ of $\mathfrak{g}$. For the magnetic weights $m \in \Lambda_{w}(\widehat{\mathrm{G}}) \subset \mathfrak{t}$ holds, while weights $\rho$ of $\mathrm{G}$ lie in the lattice $\Lambda_{w}(\mathrm{G}) \subset \mathfrak{t}^{*}$. Then, $\rho(m)$ is the dual pairing between dual spaces. Next, we interpret the contributions to the conformal dimension as closed half-spaces and hyper-planes

$$
H_{\rho}^{ \pm}=\{m \in \mathfrak{t} \mid \pm \rho(m) \geq 0\} \quad \text { and } \quad H_{\rho}=\{m \in \mathfrak{t} \mid \rho(m)=0\},
$$

for any $\rho \in \mathfrak{t}^{*}$. Define the set $\Gamma$ of relevant weights, i.e. those weights $\rho$ of G appearing in $\Delta$ for which neither $\rho$ nor $-\rho$ lies in the rational cone spanned by the simple roots $\Phi_{s}$ of G. In other words, weights $\rho$ for which the intersection of $H_{\rho}$ with $\sigma$ is not only a face of $\sigma$.

The absolute values in (1.2) are all simultaneously resolved on the rational polyhedral cones

$$
C_{\epsilon_{1}, \ldots, \epsilon_{|\Gamma|}}:=\bigcap_{\rho \in \Gamma} H_{\rho}^{\epsilon_{\rho}} \quad \text { with } \quad \epsilon_{\rho}= \pm .
$$

The action of the Weyl group $\mathcal{W}_{\mathrm{G}}$ allows us to restrict to the dominant Weyl chamber $\sigma \subset \mathfrak{t}$, which introduces a collection

$$
\tau_{\epsilon_{1}, \ldots, \epsilon_{|\Gamma|}}:=C_{\epsilon_{1}, \ldots, \epsilon_{|\Gamma|}} \cap \sigma
$$

of rational polyhedral cones inside the $\sigma$. The collection $\left\{\tau_{\left.\epsilon_{1}, \ldots, \epsilon_{|\Gamma|}\right\}}\right.$ of theses cones, which is a finite set, generates a fan $F \subset \mathfrak{t}$. Since the contributing weights are only the relevant weights, the structure of $F$ is entirely determined by the hyper-multiplet matter content. Therefore, one could call $F$ the matter fan.

As monopole operators are characterised by solutions to the generalised Dirac equation, one has to intersect the cones with the lattice $\Lambda_{w}(\widehat{\mathrm{G}})$, which yields

$$
S_{\tau}:=\tau \cap \Lambda_{w}(\widehat{\mathrm{G}}) \quad \text { for } \quad \tau \in F,
$$

a collection of additive monoids in $\Lambda_{w}(\widehat{\mathrm{G}})$. Under very reasonable assumptions, see further section 4.5 , all monoids are positive and finitely generated. For such monoids the minimal set of irreducible generators is the Hilbert basis, denoted by $\operatorname{Hilb}\left(S_{\tau}\right)$.

Outline. It is the purpose of this article to explore the implication of our approach [6] for the algebraic properties of the monopole formula and to provide a treatment for arbitrary gauge groups.

We start with a brief exposition of the geometric and algebraic view on monoids and their associated algebras in section 2. Key concepts will be triangulations of cones, refinements of fans, and free resolutions of lattice ideals. Thereafter, section 3 is devoted to the Casimir invariance and exploits the algebraic properties of invariant polynomial algebras on Lie algebras. With the collected mathematical background at hand, we explain the application to the monopole formula in section 4 . The main results lie in three different explicit expressions of the Hilbert series for the Coulomb branch of a theory with gauge group $\mathrm{G}$ and matter fan $F$. In section 5 we prove the relation between the order of the pole of $\mathrm{HS}_{\mathrm{G}}(t)$ at $t=1, t \rightarrow \infty$ with the rank of the gauge group and, consequently, the dimension of the moduli space. In addition, we provide upper bounds on the pole order for other 
roots of unity. The understanding of the Hilbert bases and the Casimir invariants allows us to identify a sufficient set of generators for the chiral ring $\mathbb{C}\left[\mathcal{M}_{C}\right]$ in section 6 . After the rather abstract considerations of the first sections, we comment on the application of the matter fan and the Hilbert bases to actual computations in section 7. Firstly, we provide a recipe on how to compute the monopole formula using computer algebra software. Secondly, we illustrate the procedure for three quiver gauge theories. In the end, section 8 concludes. For convenience of the reader, appendix A provides a reminder of employed algebro-geometric notions.

\section{Monoids and associated algebras}

\subsection{Geometric picture}

We start by exploring some known properties of cones and monoids, for details see for instance [24-27]. Let $M$ denote an $r$-dimensional real vector space and $\Lambda$ an $r$-dimensional lattice in $M$.

\subsubsection{Cones}

A rational polyhedral cone $C$ is defined as all non-negative linear combinations of a finite set of vectors $X:=\left\{v_{1}, \ldots, v_{s}\right\} \subset \Lambda$, i.e.

$$
C \equiv \operatorname{Cone}(X)=\left\{\sum_{i=1}^{s} a_{i} v_{i} \mid a_{i} \in \mathbb{R}_{\geq 0}\right\}
$$

We recall the following properties of a cone $C$ :

- $C$ is simplicial if it is generated by linearly independent vectors.

- $C$ is strongly convex or positive if $C \cap(-C)=\{0\}$.

Two concepts are important to us: faces $F<C$ of a cone $C$ and the relative interior $\operatorname{Relint}(C)$. Denote by $\mathcal{F}(C)$ the set of faces of $C$, which is a finite set containing the two improper faces: $C$ and the trivial cone $\{0\} . \mathcal{F}(C)$ is partially ordered with respect to inclusion.

Lemma 1 ([26, Cor. 2.7.6, p. 24]). Every rational polyhedral cone $C$ is the disjoint union

$$
C=\biguplus_{F \in \mathcal{F}(C)} \operatorname{Relint}(F)
$$

of the relative interiors of all its faces.

Moreover, for the strongly convex polyhedral cone $C=\operatorname{Cone}\left(v_{1}, \ldots, v_{s}\right)$ the set $X=$ $\left\{v_{1}, \ldots, v_{s}\right\}$ is a minimal generating set of $C$ if and only if $X$ contains exactly one vector $v_{E}$ from each edge $E$ of $C$ (and no other vectors). Later we will use a triangulation of a cone $C$ which is a family $\Delta$ of finitely many simplicial sub-cones such that

(i) $C=\cup_{\delta \in \Delta} \delta$, 
(ii) the faces of each $\delta \in \Delta$ are themselves members of $\Delta$, and

(iii) the intersection of each pair $\delta, \epsilon \in \Delta$ is a face of both $\delta$ and $\epsilon$.

For triangulations the following two lemmata hold:

Lemma 2 ([26, Lem. 2.11.1, p. 36]). For a simplicial cone $\delta$, generated by linearly independent vectors $x_{1}, \ldots, x_{n}$, the faces of $\delta$ are the simplicial cones Cone $(E)$, E running through the subsets of $Y:=\left\{x_{1}, \ldots, x_{n}\right\}$. There is a bijection between the set of faces $\mathcal{F}(\delta)$ and the power set $\mathcal{P}(Y)$.

Lemma 3 ([26, Lem. 2.11.2, p. 36]). Let $\Delta$ be a triangulation of the cone $C$, then the following holds:

(i) for each $\delta \in \Delta: \delta=\biguplus_{\substack{\epsilon \in \Delta \\ \epsilon \leq \delta}} \operatorname{Relint}(\epsilon)$,

(ii) $C$ is the disjoint union $C=\biguplus_{\delta \in \Delta} \operatorname{Relint}(\delta)$.

Lemma 4 ([26, Lem. 2.11.5, p. 37]). For each finitely generated cone $C$, there exists a triangulation $\Delta$ such that each simplicial sub-cone $\delta \in \Delta$ is generated by some of the generators of $C$.

\subsubsection{Monoids}

Recall that the intersection of a polyhedral cone $C \subset M$ with the lattice $\Lambda$ yields a monoid $S_{C}=C \cap \Lambda$. Many concepts applicable for cones descend to the monoid such as the following:

- The rank of a monoid $S_{C}$ equals the dimension of the cone $C$, i.e. $\operatorname{rk}\left(S_{C}\right)=\operatorname{dim}_{\mathbb{R}}(C)$.

- Let $F<C$ be a face of $C$ then $F_{S}:=F \cap S$ is called a face of $S$. The set of all faces is denoted as $\mathcal{F}(S):=\left\{F_{S} \mid F \in \mathcal{F}(C)\right\}$, which is again a finite set.

- The relative interior of a face $F_{S}$ of $S$ is defined as $F_{S}:=S \cap \operatorname{Relint}(F)=F_{S} \cap$ $\operatorname{Relint}(F)$.

- The set of invertible elements on $S$, denote by $S_{0}=\{\nu \in S \mid-\nu \in S\}$, is the largest group contained in $S$. A monoid is called positive if $S_{0}=\{0\}$. (For our purposes, $S$ is positive if it descends from a positive cone $C$.)

- For a positive monoid $S$, an element $\nu \in S$ is irreducible (in $S$ ) if $\nu=\nu_{1}+\nu_{2}$ for $\nu_{1}, \nu_{2} \in S$ is only possible if $\nu_{1}=0$ or $\nu_{2}=0$.

Lemma 5 ([28, Prop. 7.15, p. 137]). For a positive monoid $S$, the following holds:

(i) S has only finitely many irreducible elements.

(ii) S has a unique minimal generating set, given by the irreducible elements. This set is called Hilbert basis, denoted by $\operatorname{Hilb}(S)$. 
Note that the concept of a Hilbert basis relies on the positivity of the monoid as otherwise the irreducibilty is not well-defined. For our set-up, Gordan's Lemma [28, Thm. 7.16, p. 137] ensures that the monoid $S_{C}$ is finitely generated. In the proof thereof, one shows that $S_{C}$ is generated by the finite set

$$
\begin{aligned}
& \left\{v_{1}, \ldots, v_{s}\right\} \cap \operatorname{par}_{\Lambda}\left(v_{1}, \ldots, v_{s}\right) \\
\text { with } & \operatorname{par}_{\Lambda}\left(v_{1}, \ldots, v_{s}\right):=\Lambda \cap\left\{a_{1} v_{1}+\cdots+a_{s} v_{s} \mid a_{i} \in[0,1)\right\},
\end{aligned}
$$

where $\left\{v_{1}, \ldots, v_{s}\right\}$ are the minimal generators of $C$. The notion $\operatorname{par}_{\Lambda}\left(v_{1}, \ldots, v_{s}\right)$ has been initiated in [29] and stems from the underlying parallelepiped spanned by $\left\{v_{1}, \ldots, v_{s}\right\}$. Identifying the irreducible elements in $\operatorname{par}_{\Lambda}\left(v_{1}, \ldots, v_{s}\right)$ together with the cone generators yields the elements of the Hilbert basis.

Moreover, for a simplicial cone we obtain two different characterisations of $S_{C}$ : firstly, as Minkowski sum

$$
S_{C}=S_{\text {free }}+\operatorname{par}_{\Lambda}\left(v_{1}, \ldots, v_{s}\right),
$$

and, secondly, as disjoint union

$$
S_{C}=\biguplus_{x \in \operatorname{par}_{\Lambda}\left(v_{1}, \ldots, v_{s}\right)}\left(x+S_{\text {free }}\right)
$$

where $S_{\text {free }}:=\operatorname{Span}_{\mathbb{N}}\left(v_{1}, \ldots, v_{s}\right) \subset S_{C}$ is the sub-monoid (freely) generated by the cone generators $\left\{v_{1}, \ldots, v_{s}\right\}$.

\section{$2.2 \quad$ Algebraic picture}

Having introduced monoids allows to discuss their associated algebras. Let $\mathbb{K}$ be a field and $S$ a monoid in the lattice $\Lambda$. By $\mathbb{K}[S]$ we denote the monoid algebra which is a $\mathbb{K}$-vector space with basis $\boldsymbol{t}^{\nu}$, for $\nu \in S$. A generic element of $\mathbb{K}[S]$ is of the form $a_{1} \boldsymbol{t}^{\nu_{1}}+\cdots+a_{m} \boldsymbol{t}^{\nu_{m}}$ for $m \in \mathbb{N}, a_{i} \in \mathbb{K}, \nu_{i} \in S$. The additive structure of $\mathbb{K}[S]$ is clear and multiplication arises via $\boldsymbol{t}^{\nu_{1}} \cdot \boldsymbol{t}^{\nu_{2}}=\boldsymbol{t}^{\nu_{1}+\nu_{2}}$.

Lemma 6 ([27, Prop. 2.7, p. 54 \& Prop. 4.22, p. 137]). For a monoid $S$ of the lattice $\Lambda$, and $\mathbb{K}[S]$ the monoid ring (with $\mathbb{K}$ a field). The (Krull) dimension is given by $\operatorname{dim}(\mathbb{K}[S])=$ $\operatorname{rk}(S)$. In addition, the following are equivalent:

(i) $S$ is a finitely generated monoid.

(ii) $\mathbb{K}[S]$ is a finitely generated $\mathbb{K}$-algebra.

Let us briefly recall gradings of monoids, rings, and modules. Let $A$ be a monoid, then:

- An $A$-graded ring is a ring $R$, together with the decomposition $R=\bigoplus_{a \in A} R_{a}$ such that $R_{a} \cdot R_{b} \subseteq R_{a+b}$, for all $a, b \in A$. The grading is positive if the only elements of $R$ with degree $0 \in A$ are constants, i.e. $R_{0}=\mathbb{K}$ and $\mathbb{K}$ a field.

- An $A$-graded $R$-module is an $R$-module $M$, together with a decomposition $M=$ $\bigoplus_{a \in A} M_{a}$ such that $R_{a} \cdot M_{b} \subseteq M_{a+b}$ for all $a, b \in A$ 
- An $A$-graded monoid is a monoid $S$, together with a disjoint decomposition $S=$ $\biguplus_{a \in A} S_{a}$ such that $S_{a}+S_{b} \subseteq S_{a+b}$ for all $a, b \in A$. We introduce the following monoid homomorphism

$$
\phi: \begin{aligned}
& S \rightarrow A \\
& \nu \mapsto \phi(\nu)
\end{aligned} \text { such that } S_{a}=\{\nu \in S \mid \phi(\nu)=a\}
$$

which satisfies $\phi\left(\nu_{1}+\nu_{2}\right)=\phi\left(\nu_{1}\right)+\phi\left(\nu_{2}\right)$ and $\phi(n \cdot \nu)=n \cdot \phi(\nu)$ for any $n \in \mathbb{N}$ and $\nu, \nu_{1}, \nu_{2} \in S$. The grading is positive if $S_{0}=\{0\}$.

For a monoid $S$, a subset $T \subset S$ is an ideal (of $S$ ) if $S+I \subseteq I$. The radical of an ideal $I$ is $\operatorname{Rad}(I):=\{c \mid m \cdot c \in I$ for some $m \in \mathbb{N}\}$, and $I$ is a radical ideal if $I=\operatorname{Rad}(I)$. Also, a subset $T \subseteq \Lambda$ is called an $S$-module if $S+T \subseteq T$. We have the following statements:

Lemma 7 ([25, Prop. 6.1.1, p. 257]). Let $I$ be an arbitrary subset of $S: I$ is an ideal in $S$ if and only if $\mathbb{K}[I]$ is an ideal in $\mathbb{K}[S]$. A subset $T \subseteq \Lambda$ is an $S$-module if and only if the $\mathbb{K}$-vector space $\mathbb{K}(T)$, generated by $\boldsymbol{t}^{\nu}, \nu \in T$, in $\mathbb{K}[\Lambda]$ is a $\mathbb{K}[S]$-module.

This correspondence between ideal and module in $S$ and $\mathbb{K}[S]$ extends to the notion of radical ideals, prim ideals etc. Important results are (see appendix A for normality and Cohen-Macaulay)

Lemma 8 ([25, Lem. 6.1.6, p. 261]). Let $S$ be a monoid, then the ideal generated by the elements $\boldsymbol{t}^{\nu}, \nu \in \operatorname{Relint}(S)$ is a radical ideal, and is contained in every non-zero graded radical ideal of $\mathbb{K}[S]$.

Lemma 9 ([27, Cor. 2.24, p. 61]). $S_{C}=C \cap \Lambda$ is always a normal monoid, provided $C$ is finitely generated.

Lemma 10 ([25, Thm. 6.1.4, p. 260]). For $S$ a monoid and $\mathbb{K}$ a field, the following are equivalent:

(i) $S$ is a normal monoid.

(ii) $\mathbb{K}[S]$ is normal.

Lemma 11 ([25, Thm. 6.3.5, p. 272]). Let $S$ be a normal monoid and $\mathbb{K}$ a field, then

(i) $\mathbb{K}[S]$ is a Cohen-Macaulay ring and

(ii) the ideal I generated by the monomials $\boldsymbol{t}^{\nu}$ with $\nu \in \operatorname{Relint}(S)$ is the canonical module of $\mathbb{K}[S]$.

\section{$2.3 \quad$ Hilbert series}

As usual, one defines the Hilbert series for a positively $A$-graded $R$-module $M$ via

$$
\mathrm{H}_{M}(\boldsymbol{t}):=\sum_{a \in A} \mathrm{H}(M, a) \cdot \boldsymbol{t}^{a} \quad \text { with } \quad \mathrm{H}(M, a):=\operatorname{dim}_{\mathbb{K}} M_{a} .
$$


The Hilbert series for a positively $A$-graded $S$-module $T$ is defined similarly

$$
\mathrm{H}_{T}(\boldsymbol{t}):=\sum_{a \in A} \mathrm{H}(T, a) \cdot \boldsymbol{t}^{a} \quad \text { with } \quad \mathrm{H}(S, a):=\operatorname{card}\left(T_{a}\right),
$$

where $\operatorname{card}\left(T_{a}\right)$ is defined as the number of points in $T_{a}$. Note that positivity of the $A$ grading implies that both $\mathrm{H}(M, a)$ and $\mathrm{H}(S, a)$ are finite for any $a \in A$. Moreover, if follows from Lem. 7 that $\mathrm{H}_{T}(\boldsymbol{t})$ equals $\mathrm{H}_{\mathbb{K}(T)}(\boldsymbol{t})$, since $\mathbb{K}(T)$ is a $\mathbb{K}[S]$-module. There exists an alternative characterisations of (2.7a) which counts each point in the $S$-module $T$ precisely once according to their $A$-grading

$$
\mathrm{H}_{T}(\boldsymbol{t})=\sum_{\nu \in T} \boldsymbol{t}^{\phi(\nu)}
$$

This is the very same spirit as the monopole formula of [1] which counts each bare monopole operator instead of providing the Hilbert function $\mathrm{H}(\mathrm{G}, d) \equiv\{m \mid \Delta(m)=d\}$ for the Coulomb branch.

Suppose $S_{C}=C \cap \Lambda$ is the associated monoid for the cone $C=\operatorname{Cone}(X)$, with $X=\left\{v_{1}, \ldots, v_{s}\right\}$ the cone generators. We would like to compute two objects, the Hilbert series of $S_{C}$ and $\operatorname{Relint}\left(S_{C}\right)$. For this, we follow [25-27].

\subsubsection{Hilbert series for a monoid}

Let $\Delta$ be a triangulation of $C$, then define $\omega_{\delta}:=\Lambda \cap \operatorname{Relint}(\delta)$ for each $\delta \in \Delta$. The immediate consequence of Lem. 3 for the monoid $S=\Lambda \cap C$ is

$$
\mathrm{H}_{S}(\boldsymbol{t})=\sum_{\delta \in \Delta} \mathrm{H}_{\omega_{\delta}}(\boldsymbol{t}) \text {. }
$$

Since every $\delta$ is simplicial and we can choose $\Delta$ as in Lem. 4. The set of minimal generators of $\delta$ is denoted by $X_{\delta} \equiv\left\{y_{1}, \ldots, y_{r}\right\} \subset X$, a subset of the cone generators $X$ of $C$. Then $X_{\delta}$ generates a free monoid $\sigma_{\delta}=\operatorname{Span}_{\mathbb{N}}\left(X_{\delta}\right)$ whose Hilbert series reads

$$
\mathrm{H}_{\sigma_{\delta}}(\boldsymbol{t})=\frac{1}{\prod_{y \in X_{\delta}}\left(1-\boldsymbol{t}^{\phi(y)}\right)} .
$$

For $\omega_{\delta}$ we obtain the disjoint decomposition

$$
\begin{aligned}
\omega_{\delta} & =\biguplus_{x \in \operatorname{par}_{\Lambda}\left(X_{\delta}\right)}\left(x+\sigma_{\delta}\right) \\
\text { with } \operatorname{par}_{\Lambda}{ }_{\Lambda}\left(X_{\delta}\right) & :=\Lambda \cap\left\{q_{1} y_{1}+\cdots q_{r} y_{r} \mid q_{i} \in(0,1]\right\}
\end{aligned}
$$

Thus, we arrive at

$$
\mathrm{H}_{\omega_{\delta}}(\boldsymbol{t})=\frac{\sum_{x \in \operatorname{par}_{\Lambda}{ }_{\Lambda}\left(X_{\delta}\right)} \boldsymbol{t}^{\phi(x)}}{\prod_{y \in X_{\delta}}\left(1-\boldsymbol{t}^{\phi(y)}\right)} .
$$

Alternative, we rewrite $\operatorname{par}^{\prime}{ }_{\Lambda}\left(X_{\delta}\right)$ in slices $B_{a}$ of constant degree $a$, i.e.

$$
\operatorname{par}_{\Lambda}^{\prime}\left(X_{\delta}\right) \equiv \bigoplus_{a \in A_{\delta}^{\prime}} B_{a}
$$


labelled by a finite subset $A_{\delta}^{\prime} \subset A$. Rewriting (2.11) yields

$$
\mathrm{H}_{\omega_{\delta}}(\boldsymbol{t})=\frac{\sum_{a \in A_{\delta}^{\prime}} \operatorname{card}\left(B_{a}\right) \boldsymbol{t}^{a}}{\prod_{y \in X_{\delta}}\left(1-\boldsymbol{t}^{\phi(y)}\right)} .
$$

Finally, we can summarise

$$
\mathrm{H}_{S}(\boldsymbol{t})=\sum_{\delta \in \Delta} \frac{\sum_{x \in \operatorname{par}^{\prime}{ }_{\Lambda}\left(X_{\delta}\right)} \boldsymbol{t}^{\phi(x)}}{\prod_{y \in X_{\delta}}\left(1-\boldsymbol{t}^{\phi(y)}\right)}=\sum_{\delta \in \Delta} \frac{\sum_{a \in A_{\delta}^{\prime}} \operatorname{card}\left(B_{a}\right) \boldsymbol{t}^{a}}{\prod_{y \in X_{\delta}}\left(1-\boldsymbol{t}^{\phi(y)}\right)} .
$$

The two representations (2.14) are based on the two different counting arrangements of (2.7).

\subsubsection{Hilbert series for the relative interior of a monoid}

Computing the Hilbert series for the radical ideal $\operatorname{Relint}\left(S_{C}\right)$ proceeds essentially similar, but we need to modify the triangulation. Choosing the triangulation $\Delta$ of $C$ as in Lem. 4, the triangulation $\Delta$ induces also triangulations for all faces of $C$. Thus, we can define a subset

$$
\Delta^{\prime}:=\Delta \backslash\{\tau \in \Delta \mid \exists \sigma \in \mathcal{F}(C) \backslash C \text { such that } \tau \subset \sigma\} \subset \Delta,
$$

which then yields the desired property

$$
\operatorname{Relint}(C)=\biguplus_{\delta \in \Delta^{\prime}} \operatorname{Relint}(\delta) .
$$

Consequently, we obtain the Hilbert series by restriction of the results (2.14) as

$$
\mathrm{H}_{\text {Relint }(S)}(\boldsymbol{t})=\sum_{\delta \in \Delta^{\prime}} \frac{\sum_{x \in \operatorname{par}_{\Lambda}\left(X_{\delta}\right)} \boldsymbol{t}^{\phi(x)}}{\prod_{x \in X_{\delta}}\left(1-\boldsymbol{t}^{\phi(x)}\right)}=\sum_{\delta \in \Delta^{\prime}} \frac{\sum_{a \in A_{\delta}^{\prime}} \operatorname{card}\left(B_{a}\right) \boldsymbol{t}^{a}}{\prod_{x \in X_{\delta}}\left(1-\boldsymbol{t}^{\phi(x)}\right)} .
$$

\subsection{Free resolution}

Besides the pure computation of Hilbert series for monoid rings, we can additionally shed light on algebraic properties. We follow [28]. Suppose $S$ is a monoid in $\Lambda$ and $\phi: S \rightarrow A$ is the monoid morphism providing the $A$-grading of $S$. For our intends and purposes, $S$ is the monoid associated to a positive rational polyhedral cone and, hence, by Gordan's lemma is finitely generated by $\operatorname{Hilb}(S)=\left\{y_{1}, \ldots, y_{n}\right\}$. It follows that $\phi(\operatorname{Hilb}(S))$ generates the semi-group $\phi(A) \subset A$, but is not necessarily a minimal set. Consider as example $A=\mathbb{N}$ and $\phi: S \rightarrow \mathbb{N}$, but $\phi(S) \subset \mathbb{N}$ can be generated by any number $q$ of elements, for $1 \leq q \leq n$ depending on the degrees of $\phi\left(y_{i}\right)$. Another example is the grading of $S$ by itself, i.e. $A=S$ and $\phi=\left.\mathrm{id}\right|_{S}$. Then $\operatorname{Hilb}(S)$, of course, remains the minimal generating set.

Let $e_{i}$ for $i=1, \ldots, n$ be the standard basis of $\mathbb{Z}^{n}$ and define the group homomorphism

$$
\Phi: \begin{aligned}
& \mathbb{Z}^{n} \rightarrow \phi(S) \\
& e_{i} \mapsto \phi\left(y_{i}\right)
\end{aligned}
$$

The kernel $L:=\operatorname{Ker}(\Phi)$ of this map is a lattice in $\mathbb{Z}^{n}$. Consider the $A$-graded polynomial ring $R=\mathbb{K}\left[z_{1}, \ldots, z_{n}\right]$, where the grading is given by a degree map deg: $\mathbb{Z}^{n} \rightarrow A$ such 
that a monomial $\boldsymbol{z}^{u}=z_{1}^{u_{1}} \cdots z_{n}^{u_{n}}$, for $u \in \mathbb{N}^{n}$, has degree $\operatorname{deg}\left(\boldsymbol{z}^{u}\right)=\operatorname{deg}(u)=\boldsymbol{\alpha} \in A$. The distinguished set of $n$ elements $\operatorname{deg}\left(z_{1}\right), \ldots, \operatorname{deg}\left(z_{n}\right)$ in $A$ is denoted by $\boldsymbol{\alpha}_{1}, \ldots, \boldsymbol{\alpha}_{n}$.

Define the so-called lattice ideal $I_{L} \subset R$ associated to $L$ via

$$
\left.I_{L}=\left\langle\boldsymbol{z}^{u}-\boldsymbol{z}^{v}\right| u, v \in \mathbb{N}^{n} \quad \text { with } \quad u-v \in L\right\rangle .
$$

Lemma 12 ([28, Thm. 7.3, p. 130]). The A-graded monoid ring $\mathbb{K}[S]$ is isomorphic to the quotient $R / I_{L}$.

Employing the additivity of the Hilbert series in exact sequences, i.e.

$$
0 \rightarrow A \rightarrow B \rightarrow C \rightarrow 0, \text { then } \mathrm{H}_{B}=\mathrm{H}_{A}+\mathrm{H}_{C},
$$

we can deduce the Hilbert series of the monoid algebra via

$$
0 \rightarrow I_{L} \rightarrow R \rightarrow R / I_{L} \rightarrow 0, \text { hence } \mathrm{H}_{\mathbb{K}[S]}=\mathrm{H}_{R}-\mathrm{H}_{I_{L}} .
$$

Since the Hilbert series of the $A$-graded polynomial ring $R$ is simply given by

$$
\mathrm{H}_{R}(\boldsymbol{t})=\frac{1}{\prod_{i=1}^{n}\left(1-\boldsymbol{t}^{\boldsymbol{\alpha}_{i}}\right)},
$$

the question arises for the Hilbert series of the lattice ideal $I_{L}$. Here, we can employ the notion of a free resolution of an $R$-module $M$.

\subsubsection{Free resolution}

For the moment, we do not consider graded rings or modules. Recall that a free $R$-module $F$ is a direct sum $F \cong R^{r}$ for some $r \in \mathbb{N}$. A free resolution of a finitely generated $R$-module $M$ is an exact sequence of the form

$$
\cdots \rightarrow F_{i} \stackrel{f_{i}}{\longrightarrow} F_{i-1} \rightarrow \cdots \stackrel{f_{3}}{\longrightarrow} F_{2} \stackrel{f_{2}}{\longrightarrow} F_{1} \stackrel{f_{1}}{\longrightarrow} F_{0} \stackrel{f_{0}}{\longrightarrow} M \rightarrow 0
$$

between finitely generated free $R$-modules $F_{i}$. The resolution is finite if there exists some $l \in \mathbb{N}$ such that $F_{l} \neq 0$ and $F_{j}=0$ for $j>l$. If that is the case, the resolution is of length $l$ and is of the form

$$
0 \rightarrow F_{l} \stackrel{f_{l}}{\longrightarrow} F_{l-1} \rightarrow \cdots \stackrel{f_{3}}{\longrightarrow} F_{2} \stackrel{f_{2}}{\longrightarrow} F_{1} \stackrel{f_{1}}{\longrightarrow} F_{0} \stackrel{f_{0}}{\longrightarrow} M \rightarrow 0 .
$$

Lemma 13 (Hilbert Syzygy Theorem, [30, Thm. 2.1, p. 259]). Let $R=\mathbb{K}\left[y_{1}, \ldots, y_{n}\right]$. Then every finitely generated $R$-module has a finite free resolution of length at most $n$.

Benefit of a free resolution is that all appearing $R$-modules $F_{i}$ are free and, hence, the Hilbert series of each $F_{i}$ is simple to compute. Quite important, the free resolution keeps track of relations between generators of modules. To be more precise, let $M \subset R^{m}$ be an $R$-module generated by the set $F=\left\{f_{1}, \ldots, f_{s}\right\}$. The syzygy module $\operatorname{Syz}(F)$ of $F$ is the set

$$
\operatorname{Syz}(F)=\left\{\left(g_{1}, \ldots, g_{s}\right) \in R^{s} \mid f_{1} g_{1}+\cdots f_{s} g_{s}=0\right\} .
$$

An element of $\operatorname{Syz}(F)$ is called a syzygy. Put differently, the syzygy module of $F$ is the kernel of the map $R^{s} \rightarrow M$ determined by $e_{i} \mapsto f_{i}$, where $e_{i}$ is the standard basis of $R^{s}$. 


\subsubsection{Graded free resolution}

For a positively $A$-graded $R$-module we need to introduce the notion of graded homomorphisms. Let $M, N$ be graded modules over $R=\mathbb{K}\left[z_{1}, \ldots, z_{n}\right]$. A homomorphism $f: M \rightarrow N$ is a graded homomorphism of degree $\boldsymbol{\gamma} \in A$ if $f\left(M_{\boldsymbol{\alpha}}\right) \subset N_{\boldsymbol{\alpha}+\boldsymbol{\gamma}}$ for all $\boldsymbol{\alpha} \in A$. Moreover, we define the translate $M(\gamma)$ of the $R$-module $M$ by $\gamma \in A$ via the direct sum

$$
M(\gamma)=\bigoplus_{\boldsymbol{\alpha} \in A} M(\gamma)_{\boldsymbol{\alpha}} \quad \text { with } \quad M(\gamma)_{\boldsymbol{\alpha}}:=M_{\gamma+\boldsymbol{\alpha}}
$$

Then $M(d)$ is a graded $R$-module. Now, for a positively $A$-graded $R$-module $M$ a graded resolution of $M$ is a resolution of the form

$$
\cdots \rightarrow F_{2} \stackrel{f_{2}}{\longrightarrow} F_{1} \stackrel{f_{1}}{\longrightarrow} F_{0} \stackrel{f_{0}}{\longrightarrow} M \rightarrow 0
$$

where the $F_{i}$ are (twisted) free graded modules of the form $R\left(-\gamma_{i, 1}\right) \oplus \cdots \oplus R\left(-\gamma_{i, p}\right)$, and the homomorphisms between them are graded of degree 0 .

Lemma 14 (graded Hilbert Syzygy Theorem, [30, Thm. 3.8, p. 271]). Let $R=$ $\mathbb{K}\left[z_{1}, \ldots, z_{n}\right]$. Then every finitely generated graded $R$-module has a finite graded resolution of length at most $n$.

For a free $R$-module $F=R\left(-\gamma_{1}\right) \oplus \cdots \oplus R\left(-\gamma_{p}\right)$ the Hilbert series is simple to evaluate

$$
\mathrm{H}_{F}(\boldsymbol{t})=\sum_{j=1}^{p} \mathrm{H}_{R\left(-\gamma_{j}\right)}(\boldsymbol{t})=\sum_{j=1}^{p} \boldsymbol{t}^{\boldsymbol{\gamma}_{j}} \cdot \mathrm{H}_{R}(\boldsymbol{t})=\frac{\sum_{j=1}^{p} \boldsymbol{t}^{\boldsymbol{\gamma}_{j}}}{\prod_{i=1}^{n}\left(1-\boldsymbol{t}^{\boldsymbol{\alpha}_{i}}\right)} .
$$

Consequently, the Hilbert series for an $R$-module $M$ with free resolution (2.27) of length $l$ computes to

$$
\mathrm{H}_{M}(\boldsymbol{t})=\sum_{i=0}^{l}(-1)^{i} \mathrm{H}_{F_{i}}(\boldsymbol{t})=\frac{\sum_{i=0}^{l}(-1)^{i} \sum_{j=1}^{p_{i}} \boldsymbol{t}^{\boldsymbol{\gamma}_{i, j}}}{\prod_{i=1}^{n}\left(1-\boldsymbol{t}^{\boldsymbol{\alpha}_{i}}\right)} \equiv \frac{\mathcal{K}(M, \boldsymbol{t})}{\prod_{i=1}^{n}\left(1-\boldsymbol{t}^{\boldsymbol{\alpha}_{i}}\right)}
$$

wherein the $\mathcal{K}(M, \boldsymbol{t})$ denotes the so-called K-polynomial.

Ultimately, we desire to compute the Hilbert series of the quotient $\mathbb{K}[S] \cong R / I_{L}$, all we have to do is to evaluate the free resolution of $I_{L}$. The result is then given by

$$
\mathrm{H}_{\mathbb{K}[S]}(\boldsymbol{t})=\frac{1-\mathcal{K}\left(I_{L}, \boldsymbol{t}\right)}{\prod_{i=1}^{n}\left(1-\boldsymbol{t}^{\boldsymbol{\alpha}_{i}}\right)} .
$$

We will illustrate the practical application of the result later.

\subsection{Canonical module}

As introduced in Lem. 11, the relative interior of a normal monoid gives rise to the canonical module of a Cohen-Macaulay ring. The canonical module captures important properties of Cohen-Macaulay rings and is intimately related to Gorenstein rings. We refer to [27] for the details and provide the relevant points here.

First of all, a canonical module is by definition a Cohen-Macaulay module, i.e. satisfies the equality between depth and dimension, cf. appendix A. Moreover, a result by Stanley relates the Hilbert series between canonical module and Cohen-Macaulay ring: 
Lemma 15 ([27, Thm. 6.40, p. 232]). Let $S$ be a positive affine monoid and $R$ a finitely generated Cohen-Macaulay $S$-graded $\mathbb{K}$-algebra, with canonical module $\Omega_{R}$. Then

$$
\mathrm{H}_{\Omega_{R}}(\boldsymbol{t})=(-1)^{d} \mathrm{H}_{R}\left(\boldsymbol{t}^{-1}\right), \quad d=\operatorname{dim}(R) .
$$

A Cohen-Macaulay ring $R$ is Gorenstein if it is isomorphic to its own canonical module, i.e. $\Omega_{R} \cong R(a)$ for some $a \in S$. Consequently, the Hilbert series satisfies

$$
\mathrm{H}_{R}\left(\boldsymbol{t}^{-1}\right) \stackrel{\text { Lem. }}{=}{ }^{15}(-1)^{d} \mathrm{H}_{\Omega_{R}}(\boldsymbol{t})=(-1)^{d} \boldsymbol{t}^{-a} \mathrm{H}_{R}(\boldsymbol{t}),
$$

which is precisely the known criterion by Stanley [31] for $R$ to be Gorenstein. Specialising to the case of normal monoids, one obtains

Lemma 16 ([27, Thm. 6.49, p. 237]). Let $S$ be a normal affine monoid of rank d, and let $x_{1}, \ldots, x_{k}$ be the cone generators of $C_{S}=\operatorname{Span}_{\mathbb{R}_{\geq 0}}(S)$. Then

$$
\mathrm{H}_{\mathbb{K}[S]}(\boldsymbol{t})=\frac{Q(\boldsymbol{t})}{\prod_{i=1}^{k}\left(1-\boldsymbol{t}^{x_{i}}\right)}
$$

with a integer valued polynomial $Q(t)$. The Hilbert series for the ideal associated to $\operatorname{Relint}(S)$ is given by

$$
\mathrm{H}_{\mathbb{K}[\operatorname{Relint}(S)]}(\boldsymbol{t})=(-1)^{k-d} \frac{\boldsymbol{t}^{\sum_{j=1}^{k} x_{j}} Q\left(\boldsymbol{t}^{-1}\right)}{\prod_{i=1}^{k}\left(1-\boldsymbol{t}^{x_{i}}\right)} .
$$

The relation to Gorenstein rings is easily established for monoid algebras [27, Thm. 6.32, p. 226], because for a normal monoid $S, \mathbb{K}[S]$ is Gorenstein if and only if there exists $x \in S$ such that $\operatorname{Relint}(S)=x+S$. Explicitly, one observe $\mathbb{K}[\operatorname{Relint}(S)] \cong \mathbb{K}[S](x)$, such that the Hilbert series for the ideal has a palindromic numerator, since the monoid ring has.

\section{Casimir invariance}

Previously [6], we have seen that the number of dressed monopole operators for a magnetic charge $m$ is determined by the ration $\frac{P_{\mathrm{G}}(t ; m)}{P_{\mathrm{G}}(t ; 0)}$. Let us study this ratio in more detail. To start with, the classical dressing factors $P_{\mathrm{G}}(t ; m)$ had been identified with the Poincaré series $\mathrm{P}(t)$ of invariant polynomial algebras:

$$
P_{\mathrm{G}}(t ; m)=\mathrm{P}_{\mathfrak{J}\left(\mathfrak{h}_{m}\right)^{\mathcal{W}_{\mathrm{H}_{m}}}}(t)=\frac{1}{\prod_{j}\left(1-t^{b_{j}}\right)} \quad \text { and } \quad P_{\mathrm{G}}(t ; 0)=\mathrm{P}_{\mathfrak{J}(\mathfrak{h})^{\mathcal{W}_{\mathrm{G}}}}(t)=\frac{1}{\prod_{j}\left(1-t^{d_{j}}\right)},
$$

wherein $\mathfrak{h}_{m}$ is the Lie algebra of the stabiliser $\mathrm{H}_{m}$ of $m$ in $\mathrm{G}$, and $\mathcal{W}_{\mathrm{H}_{m}}$ is the Weyl group of $\mathrm{H}_{m}$. The ring $\mathfrak{J}\left(\mathfrak{h}_{m}\right)^{\mathcal{W}_{\mathrm{H}_{m}}}$ of invariants is Gorenstein for any $m$. Moreover, we know $\mathrm{T} \subseteq \mathrm{H}_{m} \subseteq \mathrm{G}$ and $\{1\} \subseteq \mathcal{W}_{\mathrm{H}_{m}} \subseteq \mathcal{W}_{\mathrm{G}}$, with $\mathrm{T}$ a maximal torus of $\mathrm{G}$ and $\mathfrak{t}=\operatorname{Lie}(\mathrm{T})$. Without loss of generality we can choose all Cartan sub-algebras $\mathfrak{h}_{m}$ and $\mathfrak{h}$ to be equal to $\mathfrak{t}$; thus, we deduce

$$
\mathfrak{J}(\mathfrak{h})^{\mathcal{W}_{\mathrm{G}}} \subset \mathfrak{J}\left(\mathfrak{h}_{m}\right)^{\mathcal{W}_{\mathrm{H}_{m}}} \subset \mathfrak{J}(\mathfrak{t})^{\{1\}} \equiv \mathfrak{P}(\mathfrak{t})
$$


Here, $\mathfrak{P}(\mathfrak{t})$ denotes the algebra of polynomials on $\mathfrak{t}$. We also know that all algebras are finitely generated by $r=\operatorname{rk}(\mathrm{G})$ algebraically independent, homogeneous elements, i.e.

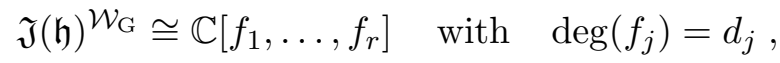

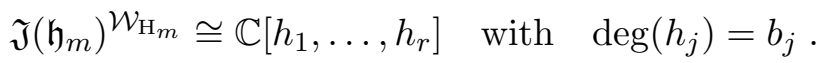

Now, consider the ideal

$$
I_{\mathrm{G}}^{\max } \equiv\left\langle f_{1}, \ldots, f_{R}\right\rangle:=\left\{\sum \chi_{j} f_{j} \mid \chi_{j} \in \mathfrak{J}(\mathfrak{h})^{\mathcal{W}_{\mathrm{G}}}\right\} \subset \mathfrak{J}(\mathfrak{h})^{\mathcal{W}_{\mathrm{G}}}
$$

which is a maximal ideal of $\mathfrak{J}(\mathfrak{h})^{\mathcal{W}_{\mathrm{G}}}$ by results of Hilbert. However, $I_{\mathrm{G}}^{\max }$ is not an ideal in $\mathfrak{J}\left(\mathfrak{h}_{m}\right)^{\mathcal{W}_{\mathrm{H}_{m}}}$, but it can be promoted to one, by considering the ideal

$$
\widetilde{I}_{\mathrm{G}}^{\max } \equiv\left\langle f_{1}, \ldots, f_{R}\right\rangle:=\left\{\sum \phi_{j} f_{j} \mid \phi_{j} \in \mathfrak{J}\left(\mathfrak{h}_{m}\right)^{\mathcal{W}_{\mathrm{H}_{m}}}\right\} \subset \mathfrak{J}\left(\mathfrak{h}_{m}\right)^{\mathcal{W}_{\mathrm{H}_{m}}}
$$

which is spanned by the same elements. Then we claim

Proposition 1. (i) The module $M_{m}^{\text {Dress }}:=\mathfrak{J}\left(\mathfrak{h}_{m}\right)^{\mathcal{W}_{\mathrm{H}_{m}}} / \widetilde{I}_{\mathrm{G}}^{\max }$ is precisely what is expected from the physical picture of the dressed monopole operators of charge $m$. That is, the bare monopole $V_{m}$ can be dressed by polynomials in the Casimir invariants of $\mathrm{H}_{m}$ which do not contain the Casimir invariant of the entire gauge group $\mathrm{G}$.

(ii) The ratio $\frac{P_{\mathrm{G}}(t ; m)}{P_{\mathrm{G}}(t ; 0)}$ of dressing factors equals the Poincaré series of the quotient $M_{m}^{\text {Dress }}$.

(iii) By our previous results [6, appendix A], we know

$$
\lim _{t \rightarrow 1} \mathrm{P}_{M_{m}^{\text {Dress }}}(t)=\frac{\left|\mathcal{W}_{\mathrm{G}}\right|}{\left|\mathcal{W}_{\mathrm{H}_{m}}\right|} \in \mathbb{N}
$$

By definition of a Poincaré series, which is morally the same as a Hilbert series (2.6), the fact (3.6) implies that $M_{m}^{\text {Dress }}$ is a finitely graded and finite dimensional module; i.e. $\operatorname{dim}\left(M_{m}^{\text {Dress }}\right)=\lim _{t \rightarrow 1} \mathrm{P}_{M_{m}^{\text {Dress }}}(t)=\sum_{i \in \mathbb{N}} \operatorname{dim}\left(\left(M_{m}^{\text {Dress }}\right)_{i}\right)<\infty$. Herein, $\left(M_{m}^{\text {Dress }}\right)_{i}$ equals the number of dressed monopole operators of charge $m$ with degree $\Delta(m)+i$.

(iv) The construction of the module $M_{m}^{\text {Dress }}$ as quotient together with the finite limit (3.6) and the definition of the Poincaré series proves that $\frac{P_{\mathrm{G}}(t ; m)}{P_{\mathrm{G}}(t ; 0)}$ is indeed an ordinary polynomial in $t$ for any $m$.

(v) $\mathrm{P}_{M_{m}^{\text {Dress }}}(t)$ is a palindromic polynomial for any $m$. The order of $\mathrm{P}_{M_{m}^{\text {Dress }}}(t)$ equals the difference in number of reflections in $\mathcal{W}_{\mathrm{G}}$ and number of reflections on $\mathcal{W}_{\mathrm{H}_{m}}$.

We start with the Poincaré series of the quotient $\mathfrak{J}\left(\mathfrak{h}_{m}\right)^{\mathcal{W}_{\mathrm{H}_{m}}} / \widetilde{I}_{\mathrm{G}}^{\max }$. Starting by induction, for $\widetilde{I}_{j} \equiv\left\langle f_{j}\right\rangle \subset \mathbb{C}\left[h_{1}, \ldots, h_{R}\right]$ we obtain the exact sequence of $\mathfrak{J}\left(\mathfrak{h}_{m}\right)^{\mathcal{W}_{\mathrm{H}_{m}} \text {-modules }}$

$$
0 \rightarrow\left\langle f_{j}\right\rangle \rightarrow \mathbb{C}\left[h_{1}, \ldots, h_{R}\right] \rightarrow \mathbb{C}\left[h_{1}, \ldots, h_{R}\right] /\left\langle f_{j}\right\rangle \rightarrow 0
$$


such that the Poincaré series behaves as

$$
\begin{gathered}
\mathrm{P}_{\mathbb{C}\left[h_{1}, \ldots, h_{R}\right]}(t)=\mathrm{P}_{\left\langle f_{j}\right\rangle}(t)+\mathrm{P}_{\mathbb{C}\left[h_{1}, \ldots, h_{R}\right] /\left\langle f_{j}\right\rangle}(t) \\
\text { with } \quad \mathrm{P}_{\mathbb{C}\left[h_{1}, \ldots, h_{R}\right]}(t)=\frac{1}{\prod_{j}\left(1-t^{b_{j}}\right)} \\
\text { and } \quad \mathrm{P}_{\left\langle f_{j}\right\rangle}(t)=t^{d_{j}} \cdot \mathrm{P}_{\mathbb{C}\left[h_{1}, \ldots, h_{R}\right]}(t) \\
\Longrightarrow \quad \mathrm{P}_{\mathbb{C}\left[h_{1}, \ldots, h_{R}\right] /\left\langle f_{j}\right\rangle}(t)=\left(1-t^{d_{j}}\right) \cdot \mathrm{P}_{\mathbb{C}\left[h_{1}, \ldots, h_{R}\right]}(t)
\end{gathered}
$$

Since the generators $\left\{f_{j}\right\}$ are algebraically independent, we can proceed iteratively and obtain the exact sequence of $\mathfrak{J}\left(\mathfrak{h}_{m}\right)^{\mathcal{W}_{\mathrm{H}_{m}} \text {-modules }}$

$$
0 \rightarrow\left\langle f_{1}, \ldots, f_{R}\right\rangle \rightarrow \mathbb{C}\left[h_{1}, \ldots, h_{R}\right] \rightarrow \mathbb{C}\left[h_{1}, \ldots, h_{R}\right] /\left\langle f_{1}, \ldots, f_{R}\right\rangle \rightarrow 0 .
$$

The Poincaré series then reduces to

$$
\mathrm{P}_{\mathbb{C}\left[h_{1}, \ldots, h_{R}\right] /\left\langle f_{1}, \ldots, f_{R}\right\rangle}(t)=\prod_{j=1}^{R}\left(1-t^{d_{j}}\right) \cdot \mathrm{P}_{\mathbb{C}\left[h_{1}, \ldots, h_{R}\right]}(t) .
$$

Employing (3.1) in (3.10) yields

$$
\mathrm{P}_{M_{m}^{\text {Dress }}}(t)=\frac{P_{\mathrm{G}}(t ; m)}{P_{\mathrm{G}}(t ; 0)}
$$

and taking the limit $t \rightarrow 1$ yields (3.6). Next, the palindromic character of $\mathrm{P}_{M_{m}^{\text {Dress }}}(t)$ is proven as follows:

$$
\begin{aligned}
& \mathrm{P}_{M_{m}^{\text {Dress }}}(t)=\frac{\prod_{i=1}^{r}\left(1-t^{d_{i}}\right)}{\prod_{j=1}^{r}\left(1-t^{b_{j}}\right)} \quad \Rightarrow \quad \mathrm{P}_{M_{m}^{\text {Dress }}}\left(\frac{1}{t}\right)=t^{-N^{-}} \mathrm{P}_{M_{m}^{\text {Dress }}}(t) \\
& \text { for } \quad N=\sum_{i=1}^{r}\left(d_{i}-b_{i}\right)=\#\left(\text { reflections in } \mathcal{W}_{\mathrm{G}}\right)-\#\left(\text { reflections in } \mathcal{W}_{\mathrm{H}_{m}}\right) \text {. }
\end{aligned}
$$

Here, we used the fact $\sum_{j=1}^{r}\left(b_{j}-1\right)=\#\left(\right.$ reflections in $\left.\mathcal{W}_{\mathrm{H}_{m}}\right)$, as stated earlier $[6$, section $2]$. At the same time, this proves that the order of $\mathrm{P}_{M_{m}^{\text {Dress }}}(t)$ equals $N$, as given in the proposition.

In addition, we can relate the set of Poincaré series $\mathrm{P}_{\mathfrak{J}\left(\mathfrak{h}_{m}\right)^{\mathcal{W}_{\mathrm{H}_{m}}}}(t)$ to another fan in the Weyl chamber $\sigma$, the set of faces $\mathcal{F}(\sigma)$.

Proposition 2. The Poincaré series $P_{\mathrm{G}}(t ; m)$ are constant along $\operatorname{Relint}\left(S_{\tau}\right)$ for all $\tau \in$ $\mathcal{F}(\sigma)$. In other words

$$
P_{\mathrm{G}}(t, m) \quad \stackrel{1: 1}{\longleftrightarrow} \quad \operatorname{Relint}\left(S_{\tau}\right), \tau \in \mathcal{F}(\sigma) .
$$

Hence, we define

$$
\mathrm{P}_{\operatorname{Relint}\left(S_{\tau}\right)}:=P_{\mathrm{G}}(t, m) \quad \text { for any } m \in \operatorname{Relint}\left(S_{\tau}\right) .
$$

The statement easily follows from recalling that the stabiliser $\mathrm{H}_{m}$ for $m \in \sigma$ depends only on the position of $m \in \operatorname{Relint}(\tau)$ for $\tau \in \mathcal{F}(\sigma)$. 


\section{Application to monopole formula}

In this section we make contact between the algebraic set-up of section 2 and 3 , and the monopole formula (1.1). Recall the set-up introduced in [6], the matter content introduces a fan $F$ within the dominant Weyl chamber $\sigma$ of the GNO-dual group $\widehat{G}$ of a semi-simple gauge group G. Moreover, the monopole formula intertwines two phenomena:

(A) Hilbert series $\mathrm{H}_{\text {Relint }\left(S_{\tau}\right)}(t)$ for the canonical module $\mathbb{K}\left[\operatorname{Relint}\left(S_{\tau}\right)\right]$ for each cone $\tau \in F$.

(B) Poincaré series $P_{\mathrm{G}}(t, m)=\mathrm{P}_{\mathfrak{J}\left(\mathfrak{h}_{m}\right)} \mathcal{W}_{\mathrm{H}_{m}}(t)$ for the $\mathrm{H}_{m}$-invariant polynomials on $\mathfrak{h}_{m} \equiv$ $\operatorname{Lie}\left(\mathrm{H}_{m}\right)$, where $\mathrm{H}_{m}=\operatorname{Stab}_{m}(G)$ denotes the residual gauge group.

The monopole formula can then be rewritten as follows:

(1) The lattice $\Lambda$ in question is the weight lattice of $\widehat{G}$. Let us emphasis this point again: the Coulomb branch is not fully specified by providing the gauge group and matter content (possibly in the form of a quiver diagram), but needs to be supplemented by the lattice of the magnetic charges. ${ }^{1}$ This lattice follows from certain boundary conditions, which translate into the generalised Dirac quantisation condition for the set-up of this article.

(2) The grading employed varies for the different monoid and is given by the conformal dimension: for $m \in S_{\tau}, \tau \in F$ we use $\operatorname{deg}(m):=\Delta(m)$. We note that this map is a monoid-homomorphism since it is a linear map on $S_{\tau}$ by construction. Furthermore, this grading is indeed a positive grading by restriction to the class of good theories, defined by [21].

(3) We can specialise the result (2.17) for a monoid $S_{\delta}$ to the relevant grading. Collecting the cone generators in the set $X_{\delta}$, one obtains

$$
\mathrm{H}_{\operatorname{Relint}\left(S_{\delta}\right)}(t)=\sum_{\delta \in \Delta^{\prime}} \frac{\sum_{s \in \operatorname{par}_{\Lambda}\left(X_{\delta}\right)} t^{\Delta(s)}}{\prod_{x \in X_{\delta}}\left(1-t^{\Delta(x)}\right)}=\sum_{\delta \in \Delta^{\prime}} \frac{\sum_{k=1}^{d_{\max }(\delta)} \operatorname{card}\left(B_{k}\right) t^{k}}{\prod_{x \in X_{\delta}}\left(1-t^{\Delta(x)}\right)}
$$

with $d_{\max }(\delta)=\sum_{x \in X_{\delta}} \Delta(x)$.

(4) By Prop. 2, the Poincaré series $P_{\mathrm{G}}(t ; m)$ is constant along $\operatorname{Relint}\left(S_{\delta}\right)$ for the face $\delta \in \mathcal{F}(\sigma)$ of the dominant Weyl chamber $\sigma$ with $m \in \operatorname{Relint}\left(S_{\delta}\right)$. The fan $F$ generated by the matter content is always a refinement of the fan $\mathcal{F}(\sigma)$; therefore, the classical dressing factor is constant along each $\operatorname{Relint}\left(S_{\tau}\right)$ for each $\tau \in F$. Hence, we define for $\tau \in F$

$$
\begin{aligned}
\mathrm{P}_{\operatorname{Relint}\left(S_{\tau}\right)}(t) & :=\mathrm{P}_{\operatorname{Relint}\left(S_{\delta}\right)}(t) \quad \text { for } \quad \delta \in \mathcal{F}(\sigma) \quad \text { with } \quad \operatorname{Relint}(\tau) \subseteq \operatorname{Relint}(\delta), \\
\text { or } \quad \mathrm{P}_{\operatorname{Relint}\left(S_{\tau}\right)}(t) & :=P_{\mathrm{G}}\left(t, m_{\text {int }}\right)
\end{aligned}
$$

\footnotetext{
${ }^{1}$ Choosing to quotient the gauge group by a discrete subgroup of its centre leads to different sub-lattices. This may lead to various possible choices of the magnetic weight lattice, in particular, when one quotients by common discrete subgroups of gauge group factors.
} 
where $m_{\text {int }}$ is any vector in $\operatorname{Relint}\left(S_{\tau}\right)$. One straightforward example can be constructed as follows: suppose $\tau=\operatorname{Cone}\left(v_{1}, \ldots, v_{s}\right)$, and $\left\{v_{1}, \ldots, v_{s}\right\} \subseteq \operatorname{Hilb}\left(S_{\tau}\right)$ is the minimal set of cone generators, which is necessarily contained in the Hilbert basis. Then one can choose $m_{\text {int }}=\sum_{i=1}^{s} v_{i} \in \operatorname{Relint} S_{\tau}$.

(5) With the aforementioned comments, we can rewrite the monopole formula of a theory with gauge group $\mathrm{G}$ and matter content defining the fan $F$ as twisted HilbertPoincaré series

$$
\begin{aligned}
\mathrm{HS}_{\mathrm{G}, F}(t) & =\sum_{\tau \in F} \mathrm{P}_{\operatorname{Relint}\left(S_{\tau}\right)}(t) \cdot \mathrm{H}_{\operatorname{Relint}\left(S_{\tau}\right)}(t) \\
& =\mathrm{P}_{\operatorname{Relint}(0)}(t) \sum_{\tau \in F} \frac{\mathrm{P}_{\operatorname{Relint}\left(S_{\tau}\right)}(t)}{\mathrm{P}_{\operatorname{Relint}(0)}(t)} \cdot \mathrm{H}_{\operatorname{Relint}\left(S_{\tau}\right)}(t) \\
& =\mathrm{P}_{\operatorname{Relint}(0)}(t) \sum_{\tau \in F} \mathrm{P}_{M_{\tau}^{\text {Dress }}(t) \cdot \mathrm{H}_{\operatorname{Relint}\left(S_{\tau}\right)}(t)}
\end{aligned}
$$

Formula (4.3a) provides a twisted sum over algebraic objects which are products of CohenMacaulay modules and Gorenstein rings. In some cases, for instance for rank one gauge groups, the monoid contributions are Gorenstein as well. The observation strongly suggests

Conjecture 1. The Coulomb branch for 3-dimensional $\mathcal{N}=4$ gauge theories is CohenMacaulay.

Although we do not have a rigorous proof, evidence for this claim stems from the structures encoded in (4.3a) and from the absence of any counter-example, see also [13].

One might wonder about the implications of Conj. 1. From the computational viewpoint, supposing that the Coulomb branch is Cohen-Macaulay allows to apply Stanley's result [31, Thm. 4.4] to check if the moduli space is even Gorenstein, purely from the Hilbert series. We emphasise that these identifications of the Coulomb branch being CohenMacaulay, Gorenstein (or even a complete intersection) are without explicitly knowing the associate coordinate ring. Moreover, by Serre's criterion for normality [25, Thm. 2.2.22, p. 71] one infers that the Coulomb branch would be a normal variety with a singular locus in codim $\geq 2$. Since the physical setting implies that the moduli space is a singular hyper-Kähler cone, the conjectured Cohen-Macaulay property would restrict the singularity. $^{2}$ From the physics point of view, the aforementioned conjecture may shed light on 3 -dimensional dualities. For instance, the Higgs branch for a 3 -dimensional $\mathcal{N}=4 \operatorname{Sp}(n)$ gauge theory with $2 n$ flavours, as considered in [32], is a union of two cones, each of which is Cohen-Macaulay, but the union is not. Then our claim in Conj. 1 on the Coulomb branch means that there cannot be a single quiver which reproduces this Higgs moduli space on the Coulomb branch side. In fact, for the Higgs branch example two quivers have been provided in [32], which generate each component. Therefore, mirror symmetry cannot be complete, as there exists a model where the mirror is not only unknown, but actually cannot exist if the above conjecture holds.

\footnotetext{
${ }^{2}$ Another "optimistical conjecture" on the Coulomb branch has been put forward in [13]: it only has symplectic singularities.
} 
Starting from (4.3) we proceed in several directions: firstly, utilising triangulations and simplicial refinements; secondly, working with free resolutions; and, lastly, combining triangulations and properties of the canonical module. The concepts will provide three different characterisations of the monopole formula. The first technique allows us to prove formal statements of the monopole formula, while the second method can be implemented in computer algebra software.

\subsection{Simplicial refinements}

From (4.1) we know how the Hilbert series for the relative interior of a monoid is computed via a triangulation. To apply this technique to the entire monopole formula (4.3) we, roughly speaking, extend the concept of triangulation to the fan $F$. To be more precise, we need to consider a simplicial refinement $\Phi$ of the fan $F$, which is a fan comprised solely of simplicial cones $\tau \in \Phi$ such that for any cone $\delta \in \Phi$ there exists at least one cone $C \in F$ such that $\delta \subseteq C$. The existence of such a refinement follows by induction over the top-dimensional cones of $F$ and their simplicial triangulations, which one chooses to be mutually compatible.

As a consequence, the refined fan $\Phi$ is a disjoint union of the relative interior of simplicial cones and, similarly to Lem. 4 , one can choose a refinement such that all simplicial cones are spanned by the cone generators of the cones in $F$. This then allows us to write $(4.3)$ as

$$
\operatorname{HS}_{\mathrm{G}, F}(t)=\mathrm{P}_{\operatorname{Relint}(0)}(t) \sum_{\tau \in \Phi} \mathrm{P}_{M_{\tau}^{\text {Dress }}}(t) \cdot \mathrm{H}_{\operatorname{Relint}\left(S_{\tau}\right)}(t),
$$

but as each cone $\tau \in \Phi$ is simplicial, we can apply (4.1) and obtain

$$
\begin{aligned}
\mathrm{HS}_{\mathrm{G}, F}(t) & =\mathrm{P}_{\text {Relint }(0)}(t) \sum_{\delta \in \Phi} \mathrm{P}_{M_{\delta}^{\text {Dress }}}(t) \cdot \frac{\sum_{k=1}^{d_{\max }(\delta)} \operatorname{card}\left(B_{k}\right) t^{k}}{\prod_{x \in X_{\delta}}\left(1-t^{\Delta(x)}\right)} \equiv \frac{R_{\mathrm{G}, F}(t)}{P_{\mathrm{G}, F}(t)} \\
R_{\mathrm{G}, F}(t) & =\sum_{\delta \in \Phi}\left\{\mathrm{P}_{M_{\delta}^{\text {Dress }}}(t) \cdot\left[\left(\sum_{k=1}^{d_{\max }(\delta)} \operatorname{card}\left(B_{k}\right) t^{k}\right) \cdot \prod_{x \in X \backslash X_{\delta}}\left(1-t^{\Delta(x)}\right)\right]\right\} \\
P_{\mathrm{G}, F}(t) & =\frac{\prod_{y \in X}\left(1-t^{\Delta(y)}\right)}{\mathrm{P}_{\operatorname{Relint}(0)}(t)}=\prod_{i=1}^{r}\left(1-t^{d_{j}}\right) \prod_{y \in X}\left(1-t^{\Delta(y)}\right)
\end{aligned}
$$

We observe that the numerator (4.4c) is a polynomial in $t$ with integer coefficients, and the denominator (4.4d) is comprised of the Casimir invariants (of degrees $d_{j}$ ) of the gauge group $\mathrm{G}$ together with the set $X$ of cone generators of $F$.

\subsection{Free resolutions}

A rather different viewpoint is taken by the use of free resolutions as introduced in section 2.4. What we are after are the relations or syzygyies between the minimal generators in a given Hilbert basis. In order to obtain their relations, based on the position in the monoid $S$ itself, we need to employ a slightly different grading at first. Again, the grading varies for the different monoids, but is now given by the graph of the conformal dimension 
that is for $m \in S_{\tau}, \tau \in F$, we use $\operatorname{deg}(m):=\Gamma_{\Delta}(m)=(m, \Delta(m))$. We note that this map is a monoid-homomorphism since it is the identity in the first factor and a linear map in the second factor. Furthermore, the grading is positive on the class of good theories, because the conformal dimension ensures that $\Gamma_{\Delta}(m)=(0,0)$ has the unique solution $m=0$.

Given this grading and having determined the Hilbert basis for each monoid $S_{\tau}, \tau \in F$, we can simply apply algorithms from algebraic geometry $[28,33]$ to compute the corresponding lattice ideals. As a consequence, the contribution of a monoid $S_{\tau}$ takes the form

$$
\mathrm{H}_{S_{\tau}}(\boldsymbol{t})=\frac{\mathcal{K}\left(S_{\tau}, \boldsymbol{t}\right)}{\prod_{x \in \operatorname{Hilb}\left(S_{\tau}\right)}\left(1-\boldsymbol{t}^{\Gamma_{\Delta}(x)}\right)} .
$$

From the previous construction (4.3), we know that we are forced to sum over the relative interiors of the monoids. As we outlined in [6], one can realise this by means of the exclusion-inclusion principle as follows

$$
\mathrm{H}_{\operatorname{Relint}\left(S_{\tau}\right)}(\boldsymbol{t})=\mathrm{H}_{S_{\tau}}(\boldsymbol{t})+\sum_{\delta \in \mathcal{F}(\tau) \backslash\{\tau\}}(-1)^{\operatorname{codim}(\delta, \tau)} \mathrm{H}_{S_{\delta}}(\boldsymbol{t})=\sum_{\delta \in \mathcal{F}(\tau)}(-1)^{\operatorname{codim}(\delta, \tau)} \mathrm{H}_{S_{\delta}}(\boldsymbol{t}),
$$

where we defined $\operatorname{codim}(\delta, \tau):=\operatorname{dim}(\tau)-\operatorname{dim}(\delta)$, and $\mathcal{F}(\tau)$ denotes the set of faces of $\tau$. One applies (4.5) to each summand and obtains

$$
\begin{aligned}
\mathrm{H}_{\text {Relint }\left(S_{\tau}\right)}(\boldsymbol{t}) & =\sum_{\delta \in \mathcal{F}(\tau)}(-1)^{\operatorname{codim}(\delta, \tau)} \frac{\mathcal{K}\left(S_{\delta}, \boldsymbol{t}\right)}{\prod_{x \in \operatorname{Hilb}\left(S_{\delta}\right)}\left(1-\boldsymbol{t}^{\Gamma_{\Delta}(x)}\right)} \\
& =\frac{\sum_{\delta \in \mathcal{F}(\tau)}\left\{(-1)^{\operatorname{codim}(\delta, \tau)} \cdot \mathcal{K}\left(S_{\delta}, \boldsymbol{t}\right) \cdot \prod_{x \in \operatorname{Hilb}\left(S_{\tau}\right) \backslash \operatorname{Hilb}\left(S_{\delta}\right)}\left(1-\boldsymbol{t}^{\Gamma_{\Delta}(x)}\right)\right\}}{\prod_{y \in \operatorname{Hilb}\left(S_{\tau}\right)}\left(1-\boldsymbol{t}^{\Gamma_{\Delta}(y)}\right)} .
\end{aligned}
$$

However, the multi-fugacity $\boldsymbol{t}$ is too much in most cases. For instance, if there are no global symmetries we only employ the $\mathrm{SU}(2)_{R}$-isospin grading. The reason why we need the monoid $\Gamma_{\Delta}\left(S_{\tau}\right)$ is to compute the lattice ideals $I_{L}$ correctly, because the syzygyies between the minimal generators are determined by the information of $S_{\tau}$ and not only the conformal dimension.

For a rank $r$ gauge group, $m$ is an $r$-dimensional vector and the multi-fugacity $\boldsymbol{t}$ consists of $r+1$ individual fugacities $t_{j}$, for $j=1, \ldots, r, r+1$. To reduce to the physically desired scenario we define

$$
\mathrm{H}_{\operatorname{Relint}\left(S_{\tau}\right)}(t):=\lim _{\substack{t_{i} \rightarrow 1 \\ i=1, \ldots, r}} \mathrm{H}_{\operatorname{Relint}\left(S_{\tau}\right)}\left(t_{1}, \ldots, t_{r}, t_{r+1} \equiv t\right)
$$

With this definition we are well-equipped to provide the full expression of the monopole 
formula

$$
\begin{aligned}
\mathrm{HS}_{\mathrm{G}, F}(t) & =\sum_{\tau \in F} \mathrm{P}_{\mathrm{Relint}\left(S_{\tau}\right)}(t) \cdot \sum_{\delta \in \mathcal{F}(\tau)}(-1)^{\operatorname{codim}(\delta, \tau)} \frac{\mathcal{K}\left(S_{\delta}, t\right)}{\prod_{x \in \operatorname{Hilb}\left(S_{\delta}\right)}\left(1-t^{\Delta(x)}\right)} \equiv \frac{\widetilde{R}_{\mathrm{G}, F}(t)}{\widetilde{P}_{\mathrm{G}, F}(t)}, \\
\widetilde{R}_{\mathrm{G}, F}(t) & =\sum_{\tau \in F} \mathrm{P}_{M_{\tau}^{\text {Dress }}}(t) \cdot \sum_{\delta \in \mathcal{F}(\tau)}\left\{(-1)^{\operatorname{codim}(\delta, \tau)} \cdot \mathcal{K}\left(S_{\delta}, t\right) \prod_{x \in \operatorname{Hilb}(F) \backslash \operatorname{Hilb}\left(S_{\delta}\right)}\left(1-t^{\Delta(x)}\right)\right\}, \\
\widetilde{P}_{\mathrm{G}, F}(t) & =\prod_{i=1}^{r}\left(1-t^{d_{j}}\right) \cdot \prod_{y \in \operatorname{Hilb}(F)}\left(1-t^{\Delta(y)}\right),
\end{aligned}
$$

where $\operatorname{Hilb}(F):=\cup_{\tau \in F} \operatorname{Hilb}\left(S_{\tau}\right)$ represents the collection of all minimal generators of the various Hilbert bases. In contrast to (4.4), the denominator $(4.9 \mathrm{c})$ is comprised of the Casimir invariants together with all monoid generators in the fan $F$. The numerator $(4.9 \mathrm{~b})$ is again a polynomial in $t$ with integer coefficients.

\subsection{Canonical module}

For completeness, we provide a third approach, which combines triangulations and the properties of canonical modules.

Suppose $\tau \in F$ with $S_{\tau}=\tau \cap \Lambda$, and let $\Delta$ be a triangulation of $\tau$. Then by combining Lem. 3, 4, and 15, one deduces

$$
\begin{aligned}
\mathrm{H}_{\text {Relint }\left(S_{\tau}\right)}(t) & =(-1)^{\mathrm{rk}\left(S_{\tau}\right)} \mathrm{H}_{S_{\tau}}\left(t^{-1}\right)=(-1)^{\mathrm{rk}\left(S_{\tau}\right)} \sum_{\delta \in \Delta} \mathrm{H}_{\operatorname{Relint}\left(S_{\delta}\right)}\left(t^{-1}\right) \\
& =\sum_{\delta \in \Delta}(-1)^{\mathrm{rk}\left(S_{\tau}\right)-\operatorname{rk}\left(S_{\delta}\right)} \mathrm{H}_{S_{\delta}}(t)=\sum_{\delta \in \Delta}(-1)^{\operatorname{dim}(\tau)-\operatorname{dim}(\delta)} \mathrm{H}_{S_{\delta}}(t),
\end{aligned}
$$

which is a result already known by Stanley [27]. Note that the Hilbert series $\mathrm{H}_{S_{\delta}}(t)$ for a monoid ring $\mathbb{K}\left[S_{\delta}\right]$ is given by (2.33a). Employing this for the monopole formula yields

$$
\begin{aligned}
\mathrm{HS}_{\mathrm{G}, F}(t) & =\sum_{\tau \in F} \mathrm{P}_{\mathrm{Relint}\left(S_{\tau}\right)}(t) \cdot \sum_{\delta \in \Delta(\tau)}(-1)^{\operatorname{codim}(\delta, \tau)} \mathrm{H}_{S_{\delta}}(t) \equiv \frac{\widehat{R}_{\mathrm{G}, F}(t)}{\widehat{P}_{\mathrm{G}, F}(t)} \\
\widehat{R}_{\mathrm{G}, F}(t) & =\sum_{\tau \in F} \mathrm{P}_{M_{\mathrm{Relint}(\tau)}^{\text {Dress }}}(t) \cdot \sum_{\delta \in \Delta(\tau)}\left\{(-1)^{\operatorname{codim}(\delta, \tau)} \cdot Q_{\delta}(t) \prod_{y \in X \backslash X_{\delta}}\left(1-t^{\Delta(y)}\right)\right\} \\
\widehat{P}_{\mathrm{G}, F}(t) & =\prod_{i=1}^{r}\left(1-t^{d_{i}}\right) \prod_{x \in X}\left(1-t^{\Delta(x)}\right)
\end{aligned}
$$

where $X_{\delta}$ denotes the cone generators of $\delta$, and $X$ the collection of all cone generators of the matter fan $F$.

Let us compare the three representations (4.4), (4.9), (4.11). Formulae (4.9) and (4.11) are exclusion-inclusion type of alternating sums. Moreover, the appearing numerators $Q$ 
and $\mathcal{K}$ are obtained from free resolutions; therefore, are integer polynomials with potentially negative signs. In contrast, formula (4.4) contains positive summands and all numerators are non-negative integer valued. Hence, this expression is most suitable for the analysis of the pole structure of section 5 .

However, the results (4.4), (4.11) employ triangulations or simplicial refinements for a further subdivision of the matter fan. Additionally, the Hilbert series for the individual monoids still have to be computed by either free resolutions of the lattice ideals or some counting algorithm for the number of points in fundamental parallelepiped. Whereas, formula (4.9) just requires the combinatorial data of the matter fan plus the free resolutions of each lattice ideal. Thus, making this approach well-suited for explicit calculations, such as present in section 7 .

Lastly, formula (4.11) is a mixture between the combinatorial and the algebraic approach. Its main feature is that it is a twisted sum of Poincaré series of normal Gorenstein rings and Hilbert series of normal Cohen-Macaulay monoid rings. The latter can in some instances be Gorenstein, too. Compared to (4.9), the denominator (4.11c) is determined by the Casimir invariants of $\mathrm{G}$ and the cone generators of $F$ in contrast to the union of all Hilbert bases.

\subsection{Comments on matter fan}

Now we comment on our construction of the matter fan described in the introduction and introduced in [6]. First of all, let us remind ourselves why we restrict to relevant weights only. Suppose $\mu$ is a weight vector of G such that $\mu \in \sigma^{\vee} \cap \Lambda_{w}(\mathrm{G})$, where $\sigma^{\vee}$ is the dual cone of the dominant Weyl chamber $\sigma$ of the GNO-dual group $\widehat{\mathrm{G}}$. Then $\sigma^{\vee}=\operatorname{Cone}\left(\alpha_{j} \mid j=\right.$ $1, \ldots, \mathrm{rk}(\mathrm{G}))$ with $\alpha_{j}$ are the simple roots of $\mathrm{G}$. By definition, $\sigma \cap H_{\mu}=: \tau_{\mu}$ defines the face $\tau_{\mu}$ of $\sigma$. Hence, $\sigma \cap H_{\mu}^{+}=\sigma$ and $\sigma \cap H_{\mu}^{-}=\tau_{\mu}$. Similarly, if $\mu$ is a weight vector for which $\mu \in\left(-\sigma^{\vee}\right) \cap \Lambda$, we see that $\operatorname{Cone}\left(\mu,\left\{\alpha_{j} \mid j=1, \ldots, \operatorname{rk}(\mathrm{G})\right\}\right)$ is not a strongly convex, because it contains the real line $\mathbb{R} \mu$. Hence, the dual cone $\operatorname{Cone}\left(\mu,\left\{\alpha_{j} \mid j=1, \ldots, \operatorname{rk}(\mathrm{G})\right\}\right)^{\vee}=$ $\bigcap_{j=1, \ldots, \mathrm{rk}(\mathrm{G})} H_{\alpha_{j}}^{+} \cap H_{\mu}^{+}$is not of maximal dimension, and since $\mu$ and $-\mu$ define the same hyper-plane, $\mu$ gives rise to the same face as $-\mu$. Therefore, the absolute value $|\mu(m)|$ is already resolved on the entire Weyl chamber $\sigma$ for weights satisfying $\pm \mu \in \sigma^{\vee} \cap \Lambda_{w}(\mathrm{G})$.

For the relevant weights, we introduced the cones (1.5). In order the define the matter fan it is sufficient to work with the top-dimensional cones, because all other cones are generated by the properties of a fan. Here, we identify the set of cones that are topdimensional and thus generate the entire matter fan. For simplicity, start with two relevant weights $\mu_{1}, \mu_{2} \in \Lambda_{w}(\mathrm{G})$. Consider the cones

$$
\begin{aligned}
\tau_{\epsilon_{1}, \epsilon_{2}} & =\bigcap_{j=1, \ldots, \mathrm{rk}(\mathrm{G})} H_{\alpha_{j}}^{+} \cap H_{\mu_{1}}^{\epsilon_{1}} \cap H_{\mu_{2}}^{\epsilon_{2}}=\bigcap_{j=1, \ldots, \mathrm{rk}(\mathrm{G})} H_{\alpha_{j}}^{+} \cap H_{\epsilon_{1} \mu_{1}}^{+} \cap H_{\epsilon_{2} \mu_{2}}^{+} \\
& =\operatorname{Cone}\left(\epsilon_{1} \mu_{1}, \epsilon_{2} \mu_{2},\left\{\alpha_{j} \mid j=1, \ldots, \operatorname{rk}(\mathrm{G})\right\}\right)^{\vee} \quad \text { with } \quad \epsilon_{i}= \pm .
\end{aligned}
$$

We distinguish the following three cases (for fixed $\epsilon_{1}$ ):

(i) For $\mu_{2} \in$ Cone $\left(\epsilon_{1} \mu_{1},\left\{\alpha_{j} \mid j=1, \ldots, \operatorname{rk}(\mathrm{G})\right\}\right), \mu_{2}$ generates a face of $\tau_{\epsilon_{1}}$, and does not further subdivide the cone $\tau_{\epsilon_{1}}$. 
(ii) For $\mu_{2} \in-$ Cone $\left(\epsilon_{1} \mu_{1},\left\{\alpha_{j} \mid j=1, \ldots, \operatorname{rk}(\mathrm{G})\right\}\right)$, Cone $\left(\epsilon_{1} \mu_{1}, \mu_{2},\left\{\alpha_{j} \mid j=1, \ldots, \operatorname{rk}(\mathrm{G})\right\}\right)$ contains the real line $\mathbb{R} \mu_{2}$. As such the dual cone is not top-dimensional, and previous arguments show that the dual cone is a proper face of $\sigma$ or the trivial cone.

(iii) For $\pm \mu_{2} \notin$ Cone $\left(\epsilon_{1} \mu_{1},\left\{\alpha_{j} \mid j=1, \ldots, \operatorname{rk}(\mathrm{G})\right\}\right)$, the two cones $\tau_{\epsilon_{1}, \epsilon_{2}}$ lead to a division of $\tau_{\epsilon_{1}}$ into two top-dimensional, strongly convex sub-cones.

For the generic case, the cones

$$
\tau_{\epsilon_{1}, \ldots, \epsilon_{|\Gamma|}}=\bigcap_{i=1, \ldots,|\Gamma|} H_{\epsilon_{i} \mu_{i}}^{+} \cap \sigma=\operatorname{Cone}\left(\left\{\epsilon_{i} \mu_{i}|i=1, \ldots,| \Gamma \mid\right\},\left\{\alpha_{j} \mid j=1, \ldots, \operatorname{rk}(G)\right\}\right)^{\vee}
$$

of (1.5) are maximal dimensional whenever $\left\{\epsilon_{i} \mu_{i}|i=1, \ldots,| \Gamma \mid\right\}$ is a necessary subset of the cone generators for the dual cone of $\tau_{\epsilon_{1}, \ldots, \epsilon|\Gamma|}$. Or in other words,

$$
\forall k \in 1, \ldots,|\Gamma|: \quad \pm \mu_{k} \notin \operatorname{Cone}\left(\left\{\epsilon_{i} \mu_{i} \mid i \neq k\right\},\left\{\alpha_{j} \mid j=1, \ldots, \operatorname{rk}(G)\right\}\right) .
$$

The resulting set of relevant, top-dimensional cones suffices to generate the fan $F$ via its defining properties: each faces of a cone in $F$ is a cone in the fan, and the intersection of two cone is a face of each.

\subsection{Product gauge groups}

So far we considered a semi-simple gauge group $\mathrm{G}$ and, hence, a semi-simple $\widehat{\mathrm{G}}$. For physically interesting theories we need to consider product groups of semi-simple $\mathrm{G}_{i}$ and abelian $\mathrm{U}(1)$ factors

$$
\prod_{i} \mathrm{G}_{i} \times \prod_{a} \mathrm{U}(1)_{a}
$$

Semi-simple gauge groups $\mathrm{G}$ are nice, because it is a priori clear that the fan $F$ consists of strongly convex cones only. Thus, the monoids are positive and the Hilbert basis is meaningful. The reason is that the dominant Weyl chamber $\sigma$ of $\widehat{G}$ is by itself a strongly convex cone and the set of faces $\mathcal{F}(\sigma)$ is understood as fan with strongly convex cones only. Since the fan $F$ is a refinement of $\mathcal{F}(\sigma)$, all cones $\tau \in F$ are strongly convex as well.

For the product $\mathrm{G}_{1} \times \mathrm{G}_{2}$ of two semi-simple groups, the GNO-dual group is $\widehat{\mathrm{G}}_{1} \times$ $\widehat{\mathrm{G}}_{2}$, by [16]. Then dominant Weyl chambers $\sigma_{1} \subset \mathbb{R}^{\mathrm{rk}\left(\mathrm{G}_{i}\right)}$ combine via the Minkowski sum into a polyhedral rational cone $\sigma_{1}+\sigma_{2} \subset \mathbb{R}^{\mathrm{rk}\left(\mathrm{G}_{1}\right)+\operatorname{rk}\left(G_{2}\right)}$. Moreover, since each $\sigma_{i}$ is strongly convex, also the sum is a strongly convex cone. Therefore, the fan $F$ is again a refinement of the set of faces of a strongly convex cone and, consequently, all cones in $F$ are strongly convex.

In the case of a product $\mathrm{U}(1) \times \mathrm{G}$, for a semi-simple $\mathrm{G}$, the dual group is still considered as $\mathrm{U}(1) \times \widehat{\mathrm{G}}$. The crux, however, lies in the appropriate notion of the dominant Weyl chamber. Since the magnetic weight lattice of $U(1)$ is $\mathbb{Z}$, we associate $\mathbb{R}$ as Weyl chamber. But then $\mathbb{R}+\sigma \subset \mathbb{R}^{\mathrm{rk}(\mathrm{G})+1}$ as combined Weyl chamber is not strongly convex any more. Fortunately, obtaining a fan $F$ containing solely strongly convex cones is achievable as long as there is any hyper-plane $H_{\mu}$ that intersects the $\mathbb{R}$ part non-trivial. This is realised in all reasonable scenarios with a contribution $|\mu(m)| \propto\left|a \cdot m_{1}+\ldots\right|$ to the conformal dimension, 
wherein $m_{1}$ is the magnetic charge in $\mathrm{U}(1)$ direction. In other words, the hyper-multiplets have to couple to the $\mathrm{U}(1)$, otherwise it would be a decoupled factor anyway. With this (mild) assumption on the hyper-plane arrangement defining $F$, the conclusion is as before: all cones in $F$ are strongly convex.

In summary, the considerations of monoid algebras, Hilbert series thereof, and applications to the monopole formula are valid for gauge groups of the form (4.15). Note that the consequences of a product gauge group for the Poincare series for the Casimir invariance for such product gauge groups has already been discussed in [6].

\section{Pole structure of the monopole formula}

Having established the generalities in section 2 and 3 , we now prove certain statements about the monopole formula that have implicitly been used in earlier works $[1,6]$.

\subsection{Order of pole at $t=1$}

Suppose we have a theory with a rank $r$ gauge group $\mathrm{G}$ and matter content defining the fan $F$. Then we claim the following:

Proposition 3. The order of the pole of the monopole formula at $t=1$ equals twice the rank of the gauge group and, thus, coincides with the complex dimension of the moduli space.

The starting point for the proof is the monopole formula (4.4), which is expressed for a simplicial refinement $\Phi$ of the fan $F$. As stated in Prop. $1, \mathrm{P}_{M_{T}^{\text {Dress }}}(t)$ is a finite polynomial with non-negative integer coefficients, i.e. a polynomial without any zeros or poles at $t=1$.

For each simplicial cone $\tau \in \Phi$, the Hilbert series of the relative interior of the corresponding monoid $S_{\tau}$ has the form (4.1). We observe that the Hilbert series (4.1) has a polynomial with non-negative integer coefficients as numerator, meaning the numerator has no zero at $t=1$. Thus, the rational function $\mathrm{H}_{\operatorname{Relint}\left(S_{\tau}\right)}(t)$ has a pole of $\operatorname{order} \operatorname{dim}(\tau)$ at $t=1$.

Putting all the pieces together in (4.4) we arrive at

$$
\mathrm{HS}_{\mathrm{G}, F}(t)=\underbrace{\mathrm{P}_{\text {Relint }(0)}(t)}_{\text {pole of order } r} \sum_{\tau \in \Phi} \underbrace{\mathrm{P}_{M_{\tau}^{\text {Dress }}(t)}}_{\text {no poles or zeros }} \cdot \underbrace{\mathrm{H}_{\text {Relint }\left(S_{\tau}\right)}(t)}_{\text {pole of order } \operatorname{dim}(\tau)} .
$$

From which we infer that the Hilbert series has a pole of order $2 r$ at $t=1$, as the maximal dimensional cones have dimension equal $r$. This argument relies on the existence of a topdimensional cone in a simplicial refinement $\Phi$. This holds, because $\Phi$ is a refinement of $F$ and, hence, also a refinement of $\mathcal{F}(\sigma)$. Since $\mathcal{F}(\sigma)$ contains $\sigma$ as the only $r$-dimensional cone, it follows that $F$ as well as $\Phi$ have at least one $r$-dimensional cone.

\subsection{Order of pole at $t \rightarrow+\infty$}

As before, consider a rank $r$ gauge group $\mathrm{G}$ and matter content defining the fan $F$, then we prove the following statement for the monopole formula: 
Proposition 4. The order of the pole at $t \rightarrow+\infty$ equals the rank of the gauge group, provided the fugacity is $t$. For fugacity $t^{2}$, the order of the pole at infinity is twice the rank of the gauge group.

Again, we start from (4.4) and the notation $X=\left\{v_{1}, \ldots, v_{r}\right\}$ for the set of all cone generators in $F$. Next, the order of the pole of $\mathrm{HS}_{\mathrm{G}, F}(t)$ at $t \rightarrow \infty$ equals the difference in maximal degrees of denominator $P_{\mathrm{G}, F}(t)$ and numerator $R_{\mathrm{G}, F}(t)$. We infer

$$
\max \operatorname{deg}\left(P_{\mathrm{G}, F}\right)=\sum_{j=1}^{r} d_{j}+\sum_{x \in X} \Delta(x)
$$

where $d_{j}$ are the degrees of the Casimir invariants of G. In addition, for each summand in the numerator $R_{\mathrm{G}, F}(t)$ we obtain

$$
\begin{aligned}
\max \operatorname{deg}\left[\left(\sum_{k=1}^{d_{\max }(\tau)}\left(\operatorname{card}\left(B_{k}\right)\right) \cdot t^{k}\right) \prod_{y \in X \backslash X_{\tau}}\left(1-t^{\Delta(y)}\right)\right] & =d_{\max }(\tau)+\sum_{y \in X \backslash X_{\tau}} \Delta(y) \\
& =\sum_{x \in X_{\tau}} \Delta(x)+\sum_{y \in X \backslash X_{\tau}} \Delta(y) \\
& =\sum_{x \in X} \Delta(x),
\end{aligned}
$$

which implies that the term in square brackets of (4.4) has always the same maximal degree, independent of the cone $\tau \in \Phi$. Then, all left to do is to find the cone with maximal degree polynomial $\mathrm{P}_{M_{\tau}^{\text {Dress }}}=\frac{P_{\operatorname{Relint}\left(S_{\tau}\right)}(t)}{P_{\operatorname{Relint}(0)}(t)}$, but this is clearly the case when the residual gauge group of $\operatorname{Relint}\left(S_{\tau}\right)$ is just the maximal torus of G. In detail, using the results from Prop. 1 or [6, appendix A] we find

$$
\max \operatorname{deg} \frac{P_{\mathrm{G}}\left(\operatorname{Relint}\left(S_{\tau}\right)\right)}{P_{\mathrm{G}}(0)}=\max \operatorname{deg} \prod_{i=1}^{r} \sum_{l_{i}}^{d_{i}-1} t^{l_{i}}=\max \operatorname{deg} \prod_{i=1}^{r} t^{d_{i}-1}=\sum_{i=1}^{r}\left(d_{i}-1\right),
$$

and finally

$$
\begin{aligned}
\max \operatorname{deg}\left(R_{\mathrm{G}, F}\right) & =\sum_{i=1}^{r}\left(d_{i}-1\right)+\sum_{x \in X} \Delta(x), \\
\max \operatorname{deg}\left(P_{\mathrm{G}, F}\right)-\max \operatorname{deg}\left(R_{\mathrm{G}, F}\right) & =\sum_{i=1}^{r} 1=r .
\end{aligned}
$$

Hence, the claim holds: the order of the pole at $t \rightarrow \infty$ equals the rank of the gauge group for fugacity $t$. Furthermore, using $t^{2}$ fugacity instead of $t$ results in overall factors of 2 in the maximal degrees of numerator and denominator. Therefore, the order of the pole is altered to $2 r$ in this case.

\subsection{Order of pole at $t=-1$}

Now, we turn to the less clear poles: to start with, let us consider $t=-1$. As before, consider a gauge group $\mathrm{G}$ of rank $r$ together with a fan $F$. Denote the collection of cone 
generators of $F$ by $X$ and let $\left\{b_{j}(\tau)\right\}_{j=1, \ldots, r}$ denote the degree of the Casimir invariants for $\mathrm{H}_{m}$ for any $m \in \operatorname{Relint}\left(S_{\tau}\right)$.

Proposition 5. The order of the pole of the Hilbert series at $t=-1$ satisfies

$$
\begin{aligned}
\text { order of pole }\left.\left(\mathrm{HS}_{\mathrm{G}, F}(t)\right)\right|_{t=-1} & \leq \max _{\tau \in F}\left\{\#\left\{b_{j}(\tau) \mid b_{j}(\tau)=\text { even }\right\}\right\} \\
& +\#\{x \in X \mid \Delta(x)=\text { even }\} \\
& \leq r+\#\{x \in X \mid \Delta(x)=\text { even }\}
\end{aligned}
$$

To prove this upper bound, we use the explicit form of (4.3) for a simplicial refinement $\Phi$ of the fan $F$ as follows:

$$
\mathrm{HS}_{\mathrm{G}, F}(t)=\sum_{\tau \in \Phi} \frac{1}{\prod_{j=1}^{r}\left(1-t^{b_{j}(\tau)}\right)} \cdot \frac{\sum_{k=1}^{d_{\max }(\tau)} \operatorname{card}\left(B_{k}\right) t^{k}}{\prod_{x \in X_{\tau}}\left(1-t^{\Delta(x)}\right)} .
$$

The order of the pole for the contribution of each cone $\tau$ can be estimated as

$$
\begin{aligned}
Z_{\tau}:=\#\left\{b_{j}(\tau) \mid b_{j}(\tau)=\text { even }\right\} & +\#\left\{x \in X_{\tau} \mid \Delta(x)=\operatorname{even}\right\} \\
& - \text { order of zero }\left\{\sum_{k=1}^{d_{\max }(\tau)} \operatorname{card}\left(B_{k}\right) t^{k} \mid t=-1\right\} .
\end{aligned}
$$

For each piece we have rough bounds

$$
\begin{aligned}
& 0 \leq \#\left\{b_{j}(\tau) \mid b_{j}(\tau)=\text { even }\right\} \leq r, \\
& 0 \leq \#\left\{x \in X_{\tau} \mid \Delta(x)=\text { even }\right\} \leq \#\{x \in X \mid \Delta(x)=\text { even }\} \\
& 0 \leq \text { order of zero }\left\{\sum_{k=1}^{d_{\max }(\tau)} \operatorname{card}\left(B_{k}\right) t^{k} \mid t=-1\right\} \leq d_{\max }(\tau)-1 .
\end{aligned}
$$

Neglecting cancellations between the summands in (5.8) we estimate an the upper bound on the order of the pole as

$$
\text { order of pole }\left.\left(\mathrm{HS}_{\mathrm{G}, F}(t)\right)\right|_{t=-1} \leq \max _{\tau \in \Phi} Z_{\tau} \text {, }
$$

after using (5.10) we obtain the claim.

\subsection{Order of pole at other roots of unity}

Lastly, we consider other roots of unity of the form $t=e^{2 \pi \mathrm{i} \frac{q}{p}}$, where we can restrict ourselves to co-prime integers $q, p$ with $0<q<p$. Consider a gauge group $\mathrm{G}$ of rank $r$ together with a fan $F$. Denote the collection of cone generators of $F$ by $X$ and let $\left\{b_{j}(\tau)\right\}_{j=1, \ldots, r}$ denote the degree of the Casimir invariants for $\mathrm{H}_{m}$ for any $m \in S_{\tau}$. 
Proposition 6. The order of the pole of the Hilbert series at $t=e^{2 \pi \mathrm{i} \frac{q}{p}}$ satisfies

$$
\begin{aligned}
\text { order of pole }\left.\left(\mathrm{HS}_{\mathrm{G}, F}(t)\right)\right|_{t=e^{2 \pi \mathrm{i}} \frac{q}{p} \leq} \max _{\tau \in F}\left\{\#\left\{b_{j}(t) \mid b_{j}(\tau) \in p \cdot \mathbb{N}\right\}\right\} \\
+\#\{x \in X \mid \Delta(x) \in p \cdot \mathbb{N}\} \\
\leq r+\#\{x \in X \mid \Delta(x) \in p \cdot \mathbb{N}\}
\end{aligned}
$$

Proving this estimates starts from (5.8) wherein the contribution of each cone is now estimated by

$$
\begin{aligned}
Z_{\tau}:=\#\left\{b_{j}(\tau) \mid b_{j}(\tau) \in p \cdot \mathbb{N}\right\} & +\#\left\{x \in X_{\tau} \mid \Delta(x) \in p \cdot \mathbb{N}\right\} \\
& - \text { order of zero }\left\{\sum_{k=1}^{d_{\max }(\tau)} \operatorname{card}\left(B_{k}\right) t^{k} \mid t=e^{2 \pi \mathrm{i} \frac{q}{p}}\right\} .
\end{aligned}
$$

The individual pieces can be restrained as

$$
\begin{aligned}
& 0 \leq \#\left\{b_{j}(\tau) \mid b_{j}(\tau) \in p \cdot \mathbb{N}\right\} \leq r \\
& 0 \leq \#\left\{x \in X_{\tau} \mid \Delta(x) \in p \cdot \mathbb{N}\right\} \leq \#\{x \in X \mid \Delta(x) \in p \cdot \mathbb{N}\} \\
& 0 \leq \text { order of zero }\left\{\sum_{k=1}^{d_{\max }(\tau)} \operatorname{card}\left(B_{k}\right) t^{k} \mid t=e^{2 \pi \mathrm{i} \frac{q}{p}}\right\} \leq \frac{d_{\max }(\tau)-1}{2} .
\end{aligned}
$$

The last estimate relies on the fact that non-real roots of unity have to appear as complex conjugated zeros of a real polynomial. Putting all the pieces together allows to derive at the desired claim.

\section{Chiral ring generators}

The introduction of fans, monoids, and Hilbert bases has not only the computational benefits for the monopole formula as Hilbert series of the Coulomb branch $\mathcal{M}_{C}$, it additionally allows us to provide a sufficient set of chiral ring generators.

Proposition 7 . The chiral ring $\mathbb{C}\left[\mathcal{M}_{C}\right]$ for a theory with rank $r$ gauge group $\mathrm{G}$ and fan $F$ is generated by the following set of dressed monopole operators:

$$
\bigcup_{x \in \operatorname{Hilb}(F)}\left\{V_{x} \cdot g \mid g \in M_{x}^{\text {Dress }}\right\} \cup\left\{V_{0} \cdot f_{j} \mid j=1, \ldots r\right\},
$$

where $\operatorname{Hilb}(F)=\cup_{\tau \in F} \operatorname{Hilb}\left(S_{\tau}\right)$ is the set of all monoid generators, and $\left\{f_{1}, \ldots, f_{r}\right\}$ are the Casimir invariants of $\mathrm{G}$. Moreover, $V_{0}$ denotes the trivial monopole operator with vanishing magnetic charge and zero conformal dimension.

To prove this claim, we proceed in steps. Firstly, notice that the (non-trivial) bare monopole operators are necessarily generated by $V_{x}$ for all $x \in \operatorname{Hilb}(F)$ by construction 


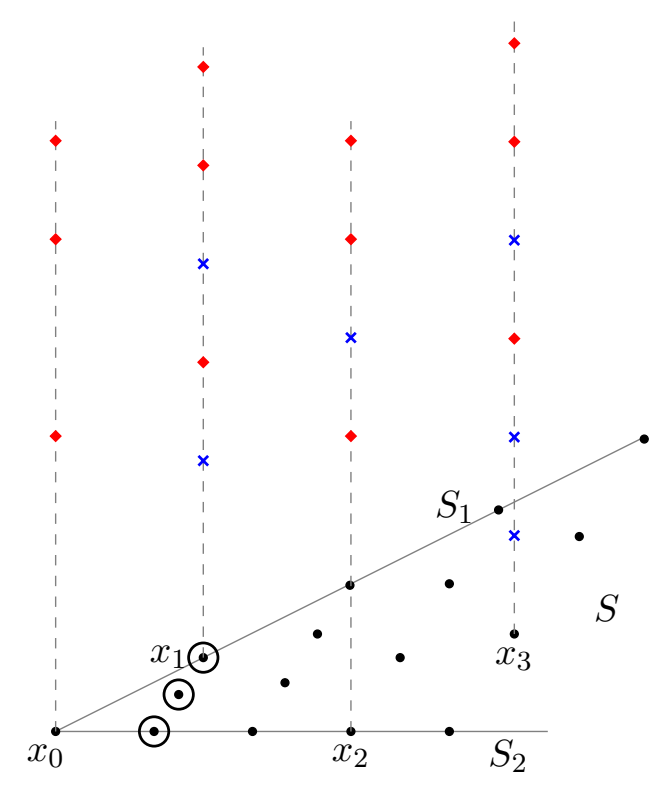

Figure 1. A rank 2 monoid $S$ with Hilbert basis given by the circled points. The "tower" over the four representative points $x_{0}, \ldots, x_{3}$ indicates the dressed monopole operators. The red points denote the dressing by Casimir invariants of the gauge group $\mathrm{G}$, while the blue crosses represent the (finite number of) dressings by the residual gauge group $\mathrm{H}_{x}$.

of the fan and the properties of the Hilbert bases. The bare monopole operators are, of course, a subset of (6.1), obtained by choosing $g=1$ in each $M_{x}^{\text {Dress }}$.

Secondly, the Casimir invariants $\left\{f_{1}, \ldots, f_{r}\right\}$ are the $r$ algebraic independent generators of $\mathfrak{J}(\mathfrak{h})^{\mathcal{W}_{\mathrm{G}}}$, which is the allowed polynomial algebra for the dressing of the origin in the magnetic weight lattice.

Thirdly, for a given bare monopole operator $V_{x}$ the algebra of dressing factors is equivalent to $\mathfrak{J}\left(\mathfrak{h}_{m}\right)^{\mathcal{W}_{\mathrm{H}_{m}}}$. Moreover, the Casimir invariants of $\mathrm{G}$ span the ideal $\widetilde{I}_{\mathrm{G}}^{\max } \subset \mathfrak{J}\left(\mathfrak{h}_{m}\right)^{\mathcal{W}_{\mathrm{H}_{m}}}$. Therefore, the only elements in $\left\{V_{x} \cdot h \mid h \in \mathfrak{J}\left(\mathfrak{h}_{m}\right)^{\mathcal{W}_{\mathrm{H}_{m}}}\right\}$ which are not generated by $V_{x} \cdot f$ for $f \in \widetilde{I}_{\mathrm{G}}^{\max }$ are precisely of the form $V_{x} \cdot g$ for $g \in M_{x}^{\text {Dress }}$. Hence, the proposition holds.

As a remark, this set of generators is really to be understood as sufficient, but in most cases it will not be necessary. That is, we have not yet identified the relations between these operators, which is still an open problem.

Lastly, let us illustrate Prop. 7 in figure 1. Displayed is a monoid $S$ of rank 2, which can be decomposed as

$$
S=\left\{x_{0}\right\} \uplus \operatorname{Relint}\left(S_{1}\right) \uplus \operatorname{Relint}\left(S_{2}\right) \uplus \operatorname{Relint}(S) .
$$

The four points originate from different parts: $x_{1} \in \operatorname{Relint}\left(S_{1}\right), x_{2} \in \operatorname{Relint}\left(S_{2}\right)$ and $x_{3} \in \operatorname{Relint}(S)$. The pile of red diamonds over $x_{0}$ denotes the dressed monopole operators of the trivial operator $V_{x_{0}}$, i.e. the Casimir invariants $\left\{f_{1}, f_{2}\right\}$ of $\mathrm{G}$ itself. In general, the degrees satisfy $\operatorname{deg}\left(f_{j}\right)>1$, such that not all (small) degrees are realised in $\mathbb{C}\left[f_{1}, f_{2}\right]$. The bare monopole operator $V_{x_{1}}$ can be dressed by the Casimir invariants of $\mathrm{G}$, which is represented by the pile of red diamonds over $x_{1}$. However, the residual gauge group is a 
subgroup of $\mathrm{G}$, such that Casimir invariance is generated by $\left\{g_{1}, g_{2}\right\}$ with different degrees as the $f_{j}$. The elements in $\mathbb{C}\left[g_{1}, g_{2}\right]$ which are not simultaneously in $\widetilde{I}_{\mathrm{G}}^{\max }$ have to lie in the quotient $M_{x_{1}}^{\text {Dress }}$. These contributions correspond to the blue crosses in the "tower" over $x_{1}$. Since the Hilbert series of $M_{x_{1}}^{\text {Dress }}$ is finite, this means that only a finite number of dressed monopole operators associated to $V_{x_{1}}$ are not expressed as product of $V_{x_{1}}$ and Casimir invariants of $\mathrm{G}$.

The very similar behaviour occurs for $x_{2}$ and $x_{3}$. Note however, that the residual gauge groups can vary even between monoids with the same rank, here $S_{1}$ and $S_{2}$. Moreover, the relative interior of $S$, which here is assumed to be a subset of the relative interior of the dominant Weyl chamber, exhibits the largest "tower" of dressed operators, as the degrees of the Casimir invariants are 1.

\section{Implementation}

In this section we show how the concepts described in the earlier sections allow for an implementation in software like Macaulay2 and Mathematica. Thus, we show how to apply the methods to more physical theories, such as certain quiver gauge theories.

\subsection{A recipe}

Given a 3 -dimensional $\mathcal{N}=4$ gauge theory with gauge group $\mathrm{G}$ and some matter content, there are five steps for computing the Hilbert series with a combined use of Macaulay2 and Mathematica. (There may be other programs capable of performing the same tasks. Here, we focus on those two.)

(I) Definition of the fan: one approach is based on the Macaulay2 package Polyhedra [34], through which cones can be defined by intersection of hyper-planes. This is precisely the approach we have advocated in [6] and elaborated on in the previous sections.

Therefore, one identifies all relevant weights in the conformal dimension of the theory and computes the hyper-planes which define the dominant Weyl chamber of the GNO-dual group. Next, the intersection of suitable subsets defines all cones, which can be grouped together as a fan $F$.

(II) Computation of the toric ideal: for each cone $\tau \in F$ one computes the Hilbert basis $\operatorname{Hilb}\left(S_{\tau}\right)$ with respect to the lattice $\Lambda \cong \mathbb{Z}^{\operatorname{rk}(G)}$ via Macaulay2.

The output $\operatorname{Hilb}\left(S_{\tau}\right)$ is graded via $\Gamma_{\Delta}$ and the resulting monoid generators are fed into the algorithm described in Algorithm for the Toric Hilbert Scheme by M. Stillman, B. Sturmfels, and R. Thomas in [33, Part II]. The output is the lattice (or toric) ideal for the corresponding monoid.

The Hilbert series for the lattice ideal as well as the quotient ring can be straight forwardly evaluated in Macaulay2. 


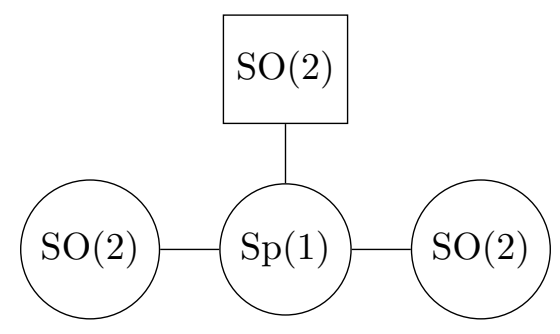

Figure 2. The quiver diagram for the first example. The magnetic charges for the two $\mathrm{SO}(2)$ factors are labelled by $n_{i} \in \mathbb{Z}$ for $i=1,2$, while the $\operatorname{Sp}(1)$ magnetic weights are denoted by $m \in \mathbb{N}$.

(III) Casimir invariance: the Poincaré series $\mathrm{P}_{\text {Relint }\left(S_{\tau}\right)}(t)$ can be evaluated as Hilbert series of the free polynomial ring $\mathbb{C}\left[f_{1}, \ldots, f_{r}\right]$ wherein one only has to define the degrees $\operatorname{deg}\left(f_{j}\right)=b_{j}$ according to the residual gauge group $\mathrm{H}_{m}$.

(IV) Combinatorics: with all the aforementioned ingredients, the final step consists of summing over all cones in the fan $F$, while for each cone $\tau$ we need to employ the exclusion-inclusion principle as shown in (4.6). Therefore, the contribution of each cone $\tau$ involves a sum over $\mathcal{F}(\tau)$.

(V) Algebraic manipulations: the Macaulay2 output can be exported into a Mathematica readable format, which allows for the algebraic manipulations such as taking the limit (4.8), bringing the sum into the form of an rational function, or analysing the result with the plethystic logarithm.

\subsection{Example quiver gauge theories}

Now, we demonstrate how our approach applies to some exemplary quiver gauge theories. We highlight in each example the geometric content of the fan and show that the sum over all cones can become cumbersome. However, the benefit is that the fan provides a structuring pattern which allows a well-defined and concise approach to the monopole formula in contrast to the pure brute force evaluation without this information.

\subsubsection{Example I: rank 3}

We study the quiver gauge theory displayed in figure 2. By the well-known fact $C_{1} \cong A_{1}$, we can treat $\mathrm{Sp}(1)$ effectively as $\mathrm{SU}(2)$. The magnetic weights of the gauge group are parametrised by $n_{j} \in \mathbb{Z}, j=1,2$, for each of the two $\mathrm{SO}(2)$ factors, and $m \in \mathbb{N}$ for the $\mathrm{Sp}(1) \cong \mathrm{SU}(2)$ factor. It readily follows that the corresponding dominant Weyl chamber $\sigma$ of the GNO-dual group is an entire half-space in $\mathbb{R}^{3}$, i.e. $\sigma$ is the $\mathbb{R}_{+}$span of the rank 3 monoid $\left\{\left(n_{1}, m, n_{2}\right) \in \mathbb{Z}^{3} \mid n_{j} \geq 0, j=1,2\right\}$. Therefore, $\sigma$ is not strongly convex cone, which is not surprising as the gauge group is not semi-simple because $\mathrm{SO}(2) \cong \mathrm{U}(1)$.

The conformal dimension of the quiver gauge theory reads

$$
\Delta_{\text {Ex.I }}\left(n_{1}, m, n_{2}\right)=\frac{1}{2} \sum_{j=1}^{2}\left(\left|n_{j}+m\right|+\left|n_{j}-m\right|\right)+|m|-2|m| .
$$




\begin{tabular}{|c|c|c|c|c|}
\hline $\operatorname{dim}(\tau)$ & 0 & 1 & 2 & 3 \\
\hline$\#(\tau), \tau \in F_{\text {Ex.I }}$ & 1 & 8 & 16 & 9 \\
\hline
\end{tabular}

Table 1. Number of cones in the $F_{\text {Ex.I }}$ for given dimension.

\begin{tabular}{|c|c|c|}
\hline \multicolumn{2}{|c|}{$\left(n_{1}, m, n_{2}, 2 \Delta\right)$} & $\mathrm{P}_{M_{\left(n_{1}, m, n_{2}\right.}^{\text {Drees }}}\left(t^{2}\right)$ \\
\hline$(1,0,0,2)$ & $(-1,0,0,2)$ & 1 \\
$(0,0,1,2)$ & $(0,0,-1,2)$ & \\
\hline$(0,1,0,2)$ & $(1,1,0,2)$ & $1+t^{2}$ \\
$(-1,1,0,2)$ & $(0,1,1,2)$ & \\
$(0,1,-1,2)$ & $(1,1,1,2)$ & \\
$(-1,1,1,2)$ & $(1,1,-1,2)$ & \\
$(-1,1,-1,2)$ & & \\
\hline \multicolumn{2}{|c|}{+2 Casimir invariants of degree 2} \\
\hline \multicolumn{2}{|c|}{+1 Casimir invariant of degree 4} \\
\hline
\end{tabular}

Table 2. The minimal generators plus their dressing behaviour of the quiver gauge theory displayed in figure 2 .

Employing this information, one defines the fan $F_{\text {Ex.I }}$ inside $\sigma$ via the following 4 hyperplanes in $\mathbb{R}^{3}$ :

$$
n_{j} \pm m=0, \quad \text { for } \quad j=1,2 .
$$

It turns out that the matter content is enough to generate a fan consisting of positive rational cones only. The number and dimensions of the cones in $F_{\text {Ex.I }}$ are provided in table 1 . The dressing factors for the gauge group $\mathrm{SO}(2) \times \mathrm{Sp}(1) \times \mathrm{SO}(2)$ are easily accounted for, because only the $\operatorname{Sp}(1)$ contribution is non-constant in $\sigma$. Thus, one obtains

$$
P_{\mathrm{Ex} . \mathrm{I}}\left(t^{2} ; n_{1}, m, n_{2}\right)=P_{\mathrm{Sp}(1)}\left(t^{2} ; m\right) \cdot \prod_{i=1}^{2} P_{\mathrm{SO}(2)}\left(t^{2} ; n_{i}\right)= \begin{cases}\frac{1}{1-t^{4}} \cdot \frac{1}{\left(1-t^{2}\right)^{2}} & m=0, \\ \frac{1}{1-t^{2}} \cdot \frac{1}{\left(1-t^{2}\right)^{2}} & m>0 .\end{cases}
$$

The collection of all Hilbert bases elements for the fan $F_{\text {Ex.I }}$ is displayed table 2. These minimal monoid generators correspond to the bare monopole operators of the theory in figure 2. After some algebraic manipulations the Hilbert series, using additional grading $z_{i}^{n_{i}}$ for the two $\mathrm{SO}(2)$ factors, reads as follows:

$$
\begin{aligned}
\mathrm{HS}_{\mathrm{Ex} . \mathrm{I}}\left(t^{2}, z_{1}, z_{2}\right)= & \frac{R_{\text {Ex.I }}\left(t^{2}, z_{1}, z_{2}\right)}{P_{\text {Ex.I }}\left(t^{2}, z_{1}, z_{2}\right)} \\
R_{\text {Ex.I }}\left(t^{2}, z_{1}, z_{2}\right)=1 & +\left(3+\frac{1}{z_{1}}+z_{1}+\frac{1}{z_{2}}+z_{2}\right) t^{2} \\
& -\left(\frac{1}{z_{1}}+z_{1}+\frac{1}{z_{2}}+z_{2}+\frac{1}{z_{1} z_{2}}+z_{1} z_{2}+\frac{z_{1}}{z_{2}}+\frac{z_{2}}{z_{1}}\right) t^{4}
\end{aligned}
$$




$$
\begin{aligned}
& -\left(\frac{1}{z_{1}}+z_{1}+\frac{1}{z_{2}}+z_{2}+\frac{1}{z_{1} z_{2}}+z_{1} z_{2}+\frac{z_{1}}{z_{2}}+\frac{z_{2}}{z_{1}}\right) t^{6} \\
& +\left(3+\frac{1}{z_{1}}+z_{1}+\frac{1}{z_{2}}+z_{2}\right) t^{8}+t^{10} \\
P_{\text {Ex.I }}\left(t^{2}, z_{1}, z_{2}\right)= & \left(1-\frac{1}{z_{1}} t^{2}\right)\left(1-z_{1} t^{2}\right)\left(1-\frac{1}{z_{2}} t^{2}\right)\left(1-z_{2} t^{2}\right) \\
& \times\left(1-\frac{1}{z_{1} z_{2}} t^{2}\right)\left(1-z_{1} z_{2} t^{2}\right)\left(1-\frac{z_{1}}{z_{2}} t^{2}\right)\left(1-\frac{z_{2}}{z_{1}} t^{2}\right) \\
\operatorname{HS}_{\text {Ex.I }}\left(t^{2}\right)= & \lim _{z_{1}, z_{2} \rightarrow 1} \operatorname{HS}_{\text {Ex.I }}\left(t^{2}, z_{1}, z_{2}\right)=\frac{1+9 t^{2}+9 t^{4}+t^{6}}{\left(1-t^{2}\right)^{6}}
\end{aligned}
$$

and subsequent expansion of the Hilbert series and the plythestic logarithm yields

$$
\begin{aligned}
\mathrm{HS}_{\mathrm{Ex} . \mathrm{I}}\left(t^{2}\right) & =1+\left(3+\frac{2}{z_{1}}+2 z_{1}+\frac{2}{z_{2}}+2 z_{2}+\frac{1}{z_{1} z_{2}}+z_{1} z_{2}+\frac{z_{1}}{z_{2}}+\frac{z_{2}}{z_{1}}\right) t^{2}+\mathcal{O}\left(t^{4}\right) \\
& \stackrel{z_{1}, z_{2} \rightarrow 1}{\longrightarrow} 1+15 t^{2}+84 t^{4}+300 t^{6}+825 t^{8}+1911 t^{10}+\mathcal{O}\left(t^{12}\right) \\
\mathrm{PL}_{\mathrm{Ex} . \mathrm{I}}\left(t^{2}\right) & =\left(3+\frac{2}{z_{1}}+2 z_{1}+\frac{2}{z_{2}}+2 z_{2}+\frac{1}{z_{1} z_{2}}+z_{1} z_{2}+\frac{z_{1}}{z_{2}}+\frac{z_{2}}{z_{1}}\right) t^{2}-\mathcal{O}\left(t^{4}\right) \\
& \stackrel{z_{1}, z_{2} \rightarrow 2}{\longrightarrow} 15 t^{2}-36 t^{4}+160 t^{6}-945 t^{8}+6048 t^{10}-\mathcal{O}\left(t^{12}\right)
\end{aligned}
$$

We readily observe the following:

(i) The Hilbert series (7.4d) has a pole of order 6 as $t$, implying that the complex dimension of the moduli space is 6 .

(ii) The difference in degrees of the denominator and the numerator of (7.4d) is 6, which agrees with complex dimension of the Coulomb branch.

(iii) The numerator is a palindromic polynomial.

(iv) The expansion (7.5a) shows that the global symmetry group of the moduli space is of dimension 15 and rank 3. Two prominent possibilities are $\mathrm{SU}(4)$ and $\mathrm{SO}(6)$, which are accidentally related via $\mathrm{SU}(4) \cong \operatorname{Spin}(6)$ and $\mathrm{SO}(6) \cong \operatorname{Spin}(6) / \mathbb{Z}_{2}$. This idea is supported by the following:

$$
\begin{aligned}
\left.\mathbf{1 5}_{\mathrm{SU}(4)}\right|_{\mathrm{SU}(3)}= & \mathbf{S U}_{\mathrm{SU}(3)}+\mathbf{3}_{\mathrm{SU}(3)}+\overline{\mathbf{3}}_{\mathrm{SU}(3)}+\mathbf{1}_{\mathrm{SU}(3)}, \\
\left.\mathrm{HS}\right|_{t^{2}}=\left.\mathrm{PL}\right|_{t^{2}}= & {\left[2+\frac{1}{z_{1}}+z_{1}+\frac{1}{z_{2}}+1 z_{2}+\frac{1}{z_{1} z_{2}}+z_{1} z_{2}\right] } \\
& +\left[z_{1}+\frac{z_{2}}{z_{1}}+\frac{1}{z_{2}}\right]+\left[z_{2}+\frac{z_{1}}{z_{2}}+\frac{1}{z_{1}}\right]+1 \\
= & \chi^{\mathrm{SU}(3)}(\mathbf{8})+\chi^{\mathrm{SU}(3)}(\mathbf{3})+\chi^{\mathrm{SU}(3)}(\overline{\mathbf{3}})+\chi^{\mathrm{SU}(3)}(\mathbf{1}) .
\end{aligned}
$$

(v) The PL (7.5b) reveals 15 generators at degree 2. These are given by the 13 minimal generators of table 2 plus the two degree 2 Casimir invariants of the gauge group. Note that the magnetic weights agree with the $z_{1}, z_{2}$-grading. 


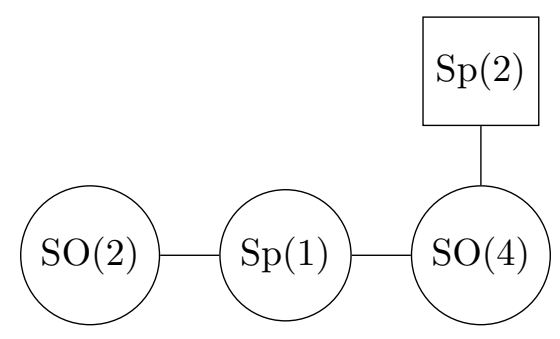

Figure 3. The quiver diagram for the second example. The magnetic charge for the $\mathrm{SO}(2)$ is labelled by $n \in \mathbb{Z}$ and for $\operatorname{Sp}(1)$ by $m \in \mathbb{N}$. The two magnetic weights of $\mathrm{SO}(4)$ are denoted by $p_{1}, p_{2} \in \mathbb{Z}$, which satisfy $p_{1} \geq\left|p_{2}\right| \geq 0$.

\begin{tabular}{|c|c|c|c|c|c|}
\hline $\operatorname{dim}(\tau)$ & 0 & 1 & 2 & 3 & 4 \\
\hline$\#(\tau), \tau \in F_{\text {Ex.II }}$ & 1 & 13 & 40 & 46 & 18 \\
\hline
\end{tabular}

Table 3. Number of cones in the fan $F_{\text {Ex.II }}$ for given dimension.

(vi) We see neither the degree 4 Casimir invariant nor the 9 dressed monopole operators of table 2 in the PL. However, it does not necessarily imply that these generators are not present.

Lastly, we note that the moduli space of the theory encoded in figure 2 is the closure of the minimal nilpotent orbit of $\mathrm{SU}(4)$ and, hence, can be compared to the study of the reduced one $\mathrm{SU}(4)$ instanton moduli space of [35].

\subsubsection{Example II: $\operatorname{rank} 4$}

Let us now examine the quiver gauge theory of figure 3. The monoid, resulting from the intersection of the dominant Weyl chamber of the GNO-dual group and the magnetic weight lattice, is parametrised via $n \in \mathbb{Z}, m \in \mathbb{N}$, and $p_{1}, p_{2} \in \mathbb{Z}$ with $p_{1} \geq\left|p_{2}\right| \geq 0$. This rank 4 monoid is non-positive due to the $\mathrm{SO}(2) \cong \mathrm{U}(1)$ factor in the gauge group. Hence, the Weyl chamber as rational cone thereof is non-positive cone in $\mathbb{R}^{4}$.

The conformal dimension of the theory under consideration reads as

$$
\begin{aligned}
\Delta_{\text {Ex.II }}\left(n, m, p_{1}, p_{2}\right)= & \frac{1}{2}(|n-m|+|n+m|) \\
& +\frac{1}{2} \sum_{j=1}^{2}\left(\left|m+p_{j}\right|+\left|m-p_{j}\right|\right)+2\left(\left|p_{1}\right|+\left|p_{2}\right|\right) \\
& -2|m|-\left|p_{1}+p_{2}\right|-\left|p_{1}-p_{2}\right| .
\end{aligned}
$$

As before, we deduce the fan $F_{\text {Ex.II }}$ via the following hyper-planes in $\mathbb{R}^{4}$ :

$$
n \pm m=0, \quad m+p_{2}=0, \quad p_{2}=0, \quad \text { and } \quad m-p_{j}=0 \quad \text { for } \quad j=1,2 .
$$

From hyper-plane arrangement we compute the fan, whose cones are summarised in table 3. Again, the matter content is enough to generate a fan that consists solely of strongly convex cones such that all monoids are positive. Because of the positivity of all monoids, we can 


\begin{tabular}{|c|c|c|}
\hline \multicolumn{2}{|c|}{$\left(n, m, p_{1}, p_{2}, 2 \Delta\right)$} & $\mathrm{P}_{M_{\left(n, m, p_{1}, p_{2}\right)}^{\text {Dress }}}\left(t^{2}\right)$ \\
\hline$(1,0,0,0,2)$ & $(-1,0,0,0,2)$ & 1 \\
\hline $\begin{array}{c}(0,1,0,0,2) \\
(-1,1,0,0,2)\end{array}$ & $(1,1,0,0,2)$ & $1+t^{2}$ \\
\hline$(0,0,1,0,2)$ & $1+2 t^{2}+t^{4}$ \\
\hline $\begin{array}{c}(0,1,1,0,2) \\
(-1,1,1,0,2)\end{array}$ & $(1,1,1,0,2)$ & $1+3 t^{2}+3 t^{4}+t^{6}$ \\
\hline \multicolumn{2}{|c|}{+1 Casimir invariant of degree 2} \\
\hline \multicolumn{2}{|c|}{+3 Casimir invariants of degree 4} \\
\hline $\begin{array}{c}(0,1,1,1,6) \\
(1,1,1,1,6)\end{array}$ & $(0,1,1,-1,6)$ & $1+2 t^{2}+t^{4}$ \\
$(1,1,1,-1,6)$ & $(-1,1,1,1,6)$ & $1+1,6)$ \\
\hline$(0,0,1,1,8)$ & $(0,0,1,-1,8)$ & $1+t^{2}$ \\
\hline
\end{tabular}

Table 4. The set of minimal generators plus their dressing behaviour of the quiver gauge theory displayed in figure 3.

compute the Hilbert bases and the corresponding dressings. The results are displayed in table 4 . In addition, the relevant classical dressing factors, which are the products of the dressing factors for each factor in the quiver gauge group, are given by

$$
P_{\mathrm{Ex} . \mathrm{II}}\left(t^{2} ; n, m, p_{1}, p_{2}\right)=P_{\mathrm{SO}(2)}\left(t^{2} ; n\right) \cdot P_{\mathrm{Sp}(1)}\left(t^{2} ; m\right) \cdot P_{\mathrm{SO}(4)}\left(t^{2} ; p_{1}, p_{2}\right) \cdot
$$

The dressing factors for the special orthogonal groups and for $\mathrm{Sp}(1) \cong \mathrm{SU}(2)$ are provided in [1]. The resulting Hilbert series, wherein we choose to additionally grade the $\mathrm{SO}(2) \cong$ $\mathrm{U}(1)$ factor by $z$, is evaluated to

$$
\mathrm{HS}_{\mathrm{Ex} . \mathrm{II}}\left(t^{2}, z\right)=\frac{1+2 t^{2}+2 t^{4}+2 t^{6}+t^{8}}{\left(1-t^{2}\right)^{2}\left(1-\frac{1}{z} t^{2}\right)^{3}\left(1-z t^{2}\right)^{3}}=\frac{\left(1-t^{4}\right)\left(1-t^{8}\right)}{\left(1-t^{2}\right)^{4}\left(1-\frac{1}{z} t^{2}\right)^{3}\left(1-z t^{2}\right)^{3}}
$$

and expanding the Hilbert series and plythestic logarithm yields

$$
\begin{aligned}
\mathrm{HS}_{\mathrm{Ex} . \mathrm{II}}\left(t^{2}, z\right)= & +\left[4+3\left(z+\frac{1}{z}\right)\right] t^{2}+\left[18+12\left(z+\frac{1}{z}\right)+6\left(z^{2}+\frac{1}{z^{2}}\right)\right] t^{4} \\
& +\left[52+45\left(z+\frac{1}{z}\right)+24\left(z^{2}+\frac{1}{z^{2}}\right)+10\left(z^{3}+\frac{1}{z^{3}}\right)\right] t^{6}+\mathcal{O}\left(t^{8}\right), \\
\mathrm{PL}_{\mathrm{Ex} . \mathrm{II}}\left(t^{2}, z\right)= & \left(4+3 \frac{1}{z}+3 z\right) t^{2}-t^{4}-t^{8} .
\end{aligned}
$$

We find the following:

(i) The Hilbert series (7.10) has a pole of order 8 at $t \rightarrow 1$, i.e. the moduli space is of complex dimension 8 . 


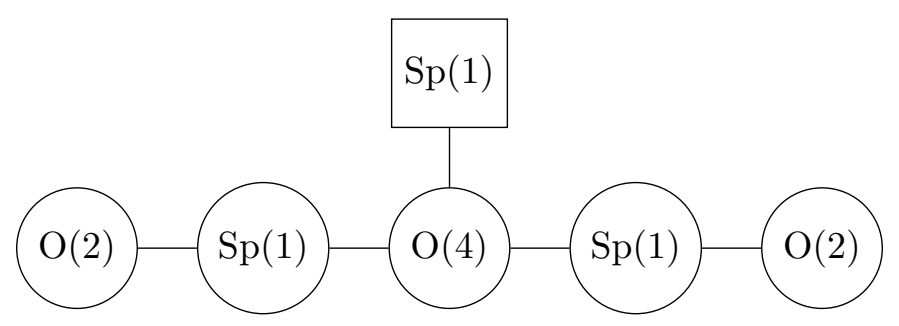

Figure 4. The quiver diagram for the third example. The magnetic charges for the $\mathrm{O}(2)$-factors are labelled by $n_{1}, n_{2} \in \mathbb{Z}$ and for the $\operatorname{Sp}(1)$-factors by $m_{1}, m_{2} \in \mathbb{N}$. The two magnetic weights of $\mathrm{O}(4)$ are denoted by $p_{1}, p_{2} \in \mathbb{N}$, which satisfy $p_{1} \geq p_{2} \geq 0$.

(ii) The difference in degrees between denominator and numerator is 8, agreeing with the dimension of the Coulomb branch as well.

(iii) The PL (7.11b) shows ten generators at degree 2, these agree with the nine minimal generators plus one Casimir invariant of table 4 . Note also that the $z$-grading agrees with the magnetic charges of the minimal generators.

(iv) The PL (7.11b) displays a relation at degree 4 as well as one relation as degree 8 . This agrees with the complete intersection form of (7.10).

Finally, let us note that the moduli space of the quiver gauge theory encoded in figure 3 is the closure of the maximal nilpotent orbit of $\mathrm{SO}(5)$.

\subsubsection{Example III: rank 6}

For the last example we choose a rank 6 quiver gauge theory as displayed in figure 4 . Let us start by commenting on some subtleties of the gauge group and its associated magnetic weight lattice. For the orthogonal groups we proceed as follows: the fundamental representation of $\mathrm{O}(2)$ stems from $\mathrm{SO}(2)$. Moreover, $\mathrm{O}(2)$ is an abelian group and as such has no contributions from vector multiplets. In contrast, the GNO-dual and the dressing factors are those of $\mathrm{SO}(3)$, as known from [5]. Next, $\mathrm{O}(4)$ behaves similarly, as the fundamental representation is inherited from $\mathrm{SO}(4)$, but the GNO magnetic weight lattice and the dressing factors are those of $\mathrm{SO}(5)$, by results of [5]. Then, the monoid describing the magnetic weights in the dominant Weyl chamber is characterised by $n_{1}, n_{2} \in$ $\mathbb{Z}, m_{1}, m_{2} \in \mathbb{N}$, and $p_{1}, p_{2} \in \mathbb{N}$ with $p_{1} \geq p_{2} \geq 0$.

The conformal dimension of this gauge theory reads

$$
\begin{aligned}
\Delta_{\text {Ex.III }}\left(n_{1}, m_{1}, p_{1}, p_{2}, m_{2}, n_{2}\right)= & \frac{1}{2} \sum_{i=1}^{2}\left(\left|m_{i}+n_{i}\right|+\left|m_{i}-n_{i}\right|\right) \\
& +\frac{1}{2} \sum_{i, j=1}^{2}\left(\left|m_{i}+p_{j}\right|+\left|m_{i}-p_{j}\right|\right)+\sum_{j=1}^{2}\left|p_{j}\right| \\
& -2 \sum_{i=1}^{2}\left|m_{i}\right|-\left|p_{1}+p_{2}\right|-\left|p_{1}-p_{2}\right| .
\end{aligned}
$$




\begin{tabular}{|c|c|c|c|c|c|c|c|}
\hline $\operatorname{dim}(\tau)$ & 0 & 1 & 2 & 3 & 4 & 5 & 6 \\
\hline$\#(\tau), \tau \in F_{\text {Ex.III }}$ & 1 & 24 & 122 & 268 & 297 & 164 & 36 \\
\hline
\end{tabular}

Table 5. Number of cones in the fan $F_{\text {Ex.III }}$ for given dimension.

We identify 5 hyper-planes in $\mathbb{R}^{6}$ intersecting the GNO Weyl chamber $\sigma$ non-trivially. These are defined via

$$
m_{i}-n_{i}=0 \quad \text { and } \quad m_{i}-p_{j}=0 \quad \text { for } \quad i=1,2, j=1,2 .
$$

Employing Macaulay2, one obtains a variety of cones that can be arranged into a fan $F_{\text {Ex.III }}$ and we summarise the cones in table 5. Similar to the previous examples, the dominant Weyl chamber of the gauge group $\mathrm{O}(2)^{2} \times \mathrm{Sp}(1)^{2} \times \mathrm{O}(4)$ is not strongly convex. However, the fan generated by the matter content consists only of strongly convex cones such that the notion of Hilbert basis is applicable to the resulting positive monoids. Hence, we computed

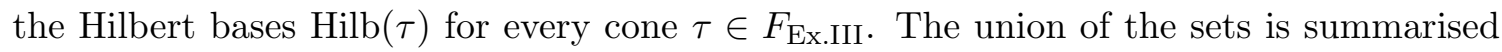
in table 6. Lastly, we need the classical dressing factors, which are the products of the dressing factors for each factor in the quiver gauge group, i.e.

$$
P_{\text {Ex.III }}\left(t^{2} ; n_{1}, m_{1}, p_{1}, p_{2}, m_{2}, n_{1}\right)=P_{\mathrm{O}(4)}\left(t^{2} ; p_{1}, p_{2}\right) \cdot \prod_{i=1}^{2} P_{\mathrm{O}(2)}\left(t^{2} ; n_{i}\right) \cdot P_{\mathrm{Sp}(1)}\left(t^{2} ; m_{i}\right) .
$$

The dressing factors for the orthogonal groups are provided in [5], and the dressing factors for $\mathrm{Sp}(1)$ are the same as for $\mathrm{SU}(2)$, see for instance [1]. We observe that the direct product structure makes it rather easy to deduces the dressing behaviour in the relative interior of a cone.

Importing the Macaulay2 output into Mathematica, we quickly arrive at

$$
\begin{aligned}
\operatorname{HS}_{\text {Ex.III }}\left(t^{2}\right)= & \frac{R_{\text {Ex.III }}\left(t^{2}\right)}{\left(1-t^{2}\right)^{6}\left(1-t^{4}\right)^{5}\left(1-t^{8}\right)} \\
R_{\text {Ex.III }}\left(t^{2}\right)= & 1+9 t^{2}+49 t^{4}+141 t^{6}+321 t^{8}+505 t^{10}+709 t^{12}+754 t^{14} \\
& +709 t^{16}+505 t^{18}+321 t^{20}+141 t^{22}+49 t^{24}+9 t^{26}+t^{28} .
\end{aligned}
$$

Let us check the propositions of section 5. The rational function (7.15) has a pole of order 12 at $t \rightarrow 1$, agreeing with the complex dimension of the Coulomb branch for the rank 6 gauge group. In addition, the numerator of (7.15) is a palindromic polynomial of degree 28 , while the denominator is a polynomial of degree 40 . Thus, the difference in degrees is 12 , coinciding with the complex dimension of the moduli space.

Next, expanding the Hilbert series (7.15) and the corresponding plythestic logarithm, we find

$$
\begin{aligned}
& \mathrm{HS}_{\text {Ex.III }}\left(t^{2}\right)=1+15 t^{2}+129 t^{4}+755 t^{6}+3462 t^{8}+13162 t^{10}+43434 t^{12}+\mathcal{O}\left(t^{14}\right) \\
& \mathrm{PL}_{\text {Ex.III }}\left(t^{2}\right)=15 t^{2}+9 t^{4}-60 t^{6}+177 t^{8}-176 t^{10}-1494 t^{12}+\mathcal{O}\left(t^{14}\right)
\end{aligned}
$$

Comparing to the Hilbert basis of table 6 and the 6 Casimir invariants of the gauge group $\mathrm{O}(2)^{2} \times \mathrm{Sp}(1)^{2} \times \mathrm{O}(4)$ of degree $4,4,4,4,4$, and 8 , we find that 


\begin{tabular}{|c|c|c|}
\hline \multicolumn{2}{|c|}{$\left(n_{1}, m_{1}, p_{1}, p_{2}, m_{2}, n_{2}, 2 \Delta\right)$} & $\mathrm{P}_{M_{\left(n_{1}, m_{1}, p_{1}, p_{2}, m_{2}, n_{2}\right)}^{\text {Dres }}}\left(t^{2}\right)$ \\
\hline$(1,0,0,0,0,0,2)$ & $(0,1,0,0,0,0,2)$ & $1+t^{2}$ \\
$(0,0,0,0,1,0,2)$ & $(0,0,0,0,0,1,2)$ & $1+2 t^{2}+t^{4}$ \\
\hline$(1,1,0,0,0,0,2)$ & $(0,0,0,0,1,1,2)$ & $1+t^{2}+t^{4}+t^{6}$ \\
\hline$(0,0,1,0,0,0,2)$ & & $1+2 t^{2}+2 t^{4}+2 t^{6}+t^{8}$ \\
\hline$(0,1,1,0,0,0,2)$ & $(0,0,1,0,1,0,2)$ & $1+3 t^{2}+4 t^{4}+4 t^{6}+3 t^{8}+t^{10}$ \\
\hline$(1,1,1,0,0,0,2)$ & $(0,1,1,0,1,0,2)$ & \\
$(0,0,1,0,1,1,2)$ & & $1+4 t^{2}+7 t^{4}+8 t^{6}+7 t^{8}+4 t^{10}+t^{12}$ \\
\hline$(1,1,1,0,1,0,2)$ & $(0,1,1,0,1,1,2)$ & $1+3 t^{2}+4 t^{4}+4 t^{6}+3 t^{8}+t^{10}$ \\
\hline$(1,1,1,0,1,1,2)$ & & $1+4 t^{2}+7 t^{4}+8 t^{6}+7 t^{8}+4 t^{10}+t^{12}$ \\
\hline$(0,1,1,1,1,0,4)$ & & $11 t^{4}+15 t^{6}+15 t^{8}+11 t^{10}+5 t^{12}+11 t^{4}+15 t^{6}+15 t^{8}+11 t^{10}+5 t^{12}+t^{14}$ \\
\hline$(1,1,1,1,1,0,4)$ & $(0,1,1,1,1,1,4)$ & $1+2 t^{2}+2 t^{4}+2 t^{6}+t^{8}$ \\
\hline$(1,1,1,1,1,1,4)$ & & $1+3 t^{2}+4 t^{4}+4 t^{6}+3 t^{8}+t^{10}$ \\
\hline \multicolumn{3}{|c|}{+5 Casimir invariants of degree 4} \\
\hline$(0,1,1,1,0,0,6)$ & $(0,0,1,1,1,0,6)$ & $1+t^{2}+t^{4}+t^{6}$ \\
\hline$(0,0,1,1,1,1,6)$ & $(1,1,1,1,0,0,6)$ & \\
\hline$(0,0,1,1,0,0,8)$ & & \\
\hline \multicolumn{2}{|c|}{+1 Casimir invariant of degree 8} \\
\hline
\end{tabular}

Table 6. All minimal generators for the monoids associated to the cones in the fan $F_{\text {Ex.III }}$. Note that we get exactly 24 generators, which equals the number of rays in $F_{\text {Ex.III }}$. Therefore, each Hilbert basis $\operatorname{Hilb}\left(S_{\tau}\right)$ coincides with the corresponding cone basis of $\tau$.

(i) $\left(1-t^{4}\right)^{5}\left(1-t^{8}\right)$ of $(7.15)$ may be chosen to correspond to the Casimir invariants of the gauge group.

(ii) The coefficient 15 of the $t^{2}$ in the PL correspond to the fifteen degree 2 minimal generators of table 6 .

(iii) While the coefficient 9 of the $t^{4}$ in the PL correspond to the five degree 4 Casimir invariants plus the four degree 4 generators of table 6 .

(iv) Thus, we are only missing the four degree 6 and the one degree 8 generators of table 6 , as well as the degree 8 Casimir invariant of the gauge group factor $\mathrm{O}(4)$.

(v) The $t^{2}$ coefficient in expansion of the Hilbert series suggest that the global symmetry group of the moduli space is of dimension 15, which would suggest $\mathrm{SO}(6)$ and $\mathrm{SU}(4)$. Both options are related via $\mathrm{SU}(4) \cong \operatorname{Spin}(6)$ and $\operatorname{Spin}(6) / \mathbb{Z}_{2} \cong \mathrm{SO}(6)$.

In contrast to the previous two examples, this moduli space is not a nilpotent orbit. For details on those orbits see for instance [36]. 


\section{Conclusions}

In this article we have explored the implications of understanding the monopole formula as being organised by a fan $F$ and its associated collection of monoids $S_{\tau}, \tau \in F$. From the mathematical point of view this approach is very rich as additive monoids and their associated algebras are well-studied. Consequently, we have reformulated the entire monopole formula in four ways:

(i) As twisted sum (4.3) of Hilbert series for modules corresponding to $\operatorname{Relint}\left(S_{\tau}\right)$ times Poincaré series for the Casimir invariance along $\operatorname{Relint}\left(S_{\tau}\right)$. This form connects the monopole formula with two mathematically well-defined objects.

(ii) As explicit rational function (4.4) by utilising a simplicial refinement of the matter fan $F$. Here, the Casimir invariants of $\mathrm{G}$ together with the cone generators of $F$ determine the denominator. Moreover, this form is particularly useful to prove the pole order statements of Prop. $3-6$ for $t^{n}=1$ and $t \rightarrow \infty$.

(iii) As explicit rational function (4.9) by means of free resolutions of the lattice ideals for each monoid. The denominator is determined by the Casimir invariants of $\mathrm{G}$ and the Hilbert bases of $F$. In addition, we found the approach well-suited for the evaluation of the monopole formula with computer algebra systems.

(iv) As explicit rational function (4.11) by merging triangulations with the properties of canonical modules. Again, the Casimir invariants of $\mathrm{G}$ together with the cone generators of $F$ provide the denominator.

The appearing structures and the wealth of known examples strongly suggest that the Coulomb branches are Cohen-Macaulay, which is the content of Conj. 1.

We have provided further insides in the dressing behaviour of monopole operators in Prop. 1-2 and reduced the effects for an operator of magnetic charge $x$ to the module $M_{x}^{\text {Dress }}$. The Poincaré series for this module equals the ratio of dressing factors $P_{\mathrm{G}}(t ; x) /$ $P_{\mathrm{G}}(t ; 0)$. On the one hand, it demonstrates that only a finite number of dressed monopole generators for each $x$ exist. On the other hand, it allows to identify a sufficient set of chiral ring generators as in Prop. 7.

Lastly, we have computed the Hilbert series for three quiver gauge theories of higher rank using the approach advocated before. In each case, the fan serves as ordering scheme to an otherwise very cumbersome task. This demonstrates that the novel view point is not limited to the rank 2 case of our earlier work [6].

Before closing, we comment on open questions that we would like to address in the future. The identification of the chiral ring generators is a first step, but we really need to understand the relations between them. There is a precise notion of relations/syzygies encoded in the lattice ideal and its resolution. However, it is at the moment not clear to us whether they are related to the relations on the Coulomb branch itself. Additionally, it would be interesting to see if the twisted structure of the Hilbert series and the local product structure $\mathfrak{J}\left(\mathfrak{h}_{\mathrm{H}_{x}}\right)^{\mathcal{W}_{\mathrm{H}_{x}}} \times \mathbb{K}\left[\operatorname{Relint}\left(S_{\tau}\right)\right]$ (for $x \in \operatorname{Relint}\left(S_{\tau}\right)$ ) allow a global understanding of the Coulomb branch. 


\section{Acknowledgments}

We thank Simon Brandhorst, Santiago Cabrera, Bo Feng, Giulia Ferlito, Yang-Hui He, Rudolph Kalveks, and Zhenghao Zhong for useful discussions. A. H. is supported by STFC Consolidated Grant STJ00035331, and EPSRC Programme Grant EP/K034456/1. M. S. was supported by the DFG research training group GRK1463 "Analysis, Geometry, and String Theory" and the Institut für Theoretische Physik of the Leibniz Universität Hannover. M. S. is currently supported by Austrian Science Fund (FWF) grant P28590.

\section{A Reminder: algebraic geometry}

In this appendix we provide the definitions and examples for algebraic concepts we have used in the main text. In particular, we elaborate on Cohen-Macaulay and Gorenstein rings. Roughly speaking, these are rings with nice properties compared to a generic ring and have shown their (mathematical and physical) relevance in the study of toric varieties, cf. [37]. For instance, normal toric varietes are Cohen-Macaulay, and are in addition Gorenstein if a certain condition on the canonical divisor is satisfied. For this exposition, we follow [25] and refer to standard textbooks for a more detailed treatment.

Besides the (Krull) dimension, the depth is another important numerical invariant of a ring $^{3} R$ or a finite $R$-module $M$. We now recall the definition: an element $x \in R$ is called $M$-regular if $x z=0$ for $z \in M$ implies $z=0$. A sequence $\boldsymbol{x}=x_{1}, \ldots, x_{n}$ of elements in $R$ is called an $M$-regular sequence if two conditions hold: (i) $x_{i}$ is an $M /\left\langle x_{1}, \ldots, x_{i-1}\right\rangle$ regular element for $i=1, \ldots, n$, and (ii) $M /\langle\boldsymbol{x}\rangle \neq 0$. As example, consider the sequence $z_{1}, \ldots, z_{k}$ of variables of $R=\mathbb{K}\left[z_{1}, \ldots, z_{k}\right]$. The first condition reads: $z_{i}$ is regular in $R /$ $\left\langle z_{1}, \ldots, z_{i-1}\right\rangle \cong \mathbb{K}\left[z_{i}, \ldots, z_{k}\right]$; while the second condition yields the field over which $R$ is defined, i.e. $R /\left\langle z_{1}, \ldots, z_{k}\right\rangle \cong \mathbb{K}$. Hence, $z_{1}, \ldots, z_{k}$ is an $R$-regular sequence.

Given an $M$-sequence $\boldsymbol{x}=x_{1}, \ldots, x_{n}$, one observes that the sequence

$$
\left\langle x_{1}\right\rangle \subset\left\langle x_{1}, x_{2}\right\rangle \subset \ldots \subset\left\langle x_{1}, \ldots, x_{n}\right\rangle
$$

is strictly ascending. An $M$-sequence can be extended to a maximal such sequence, i.e. an $M$-sequence $\boldsymbol{x}$ is maximal if $x_{1}, \ldots, x_{n+1}$ is not an $M$-sequence for any $x_{n+1} \in R$. An important fact is that all maximal $M$-sequences in an ideal $I$ of $R$ with $I M \neq M$ have the same length, called grade, provided $M$ is finite [25, Thm. 1.2.5, p. 10]. The depth of $M$ is then defined as the grade of a maximal ideal $\mathfrak{m}$ of $R$. The relation between depth and dimension of an $R$-module $M$ is $\operatorname{depth}(M) \leq \operatorname{dim}(M)$, cf. [25, Prop. 1.2.12, p. 12].

With the depth at hand, one defines that a finite $R$-module $M \neq 0$ is a CohenMacaulay module if $\operatorname{depth}(M)=\operatorname{dim}(M)$. If $R$ itself is a Cohen-Macaulay module, then it is called a Cohen-Macaulay ring. As we know from Lem. 10 and 11, a monoid ring $\mathbb{K}[S]$ is Cohen-Macaulay if and only if $S$ is a normal monoid.

We recall that the normalisation of $R$ in the $R$-module $M$ is the ring if all elements of $M$ which are integral over $R$. For the special case of $M$ being the quotient ring of an

\footnotetext{
${ }^{3}$ To be precise, one considers a Noetherian local ring, which we from now on assume.
} 


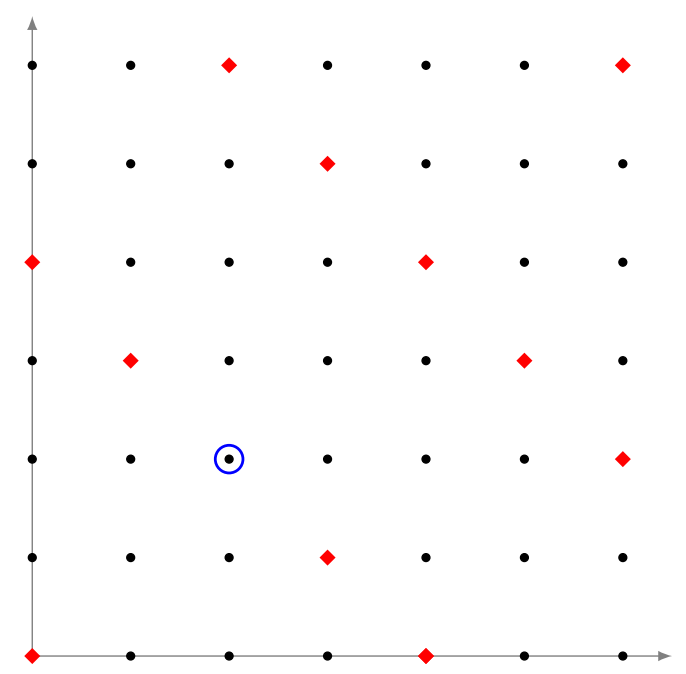

Figure 5. The monoid $S_{1}$ spanned by $\{(4,0),(3,1),(1,3),(0,4)\}$ is drawn by red diamonds, while red diamonds and black dots together form $\mathbb{Z}^{2}$. The blue circled point is $(2,2)$.

integral domain $R$ it is simply called the normalisation of $R$. A integral ring is called normal if it is own normalisation. For monoid rings $\mathbb{K}[S]$, the normality becomes a condition on the underlying monoid by Lem. 10. Let $S$ be a monoid in the lattice $\Lambda$, an element $\nu \in \Lambda$ is integral over $S$ if $c \nu \in S$ for some $c \in \mathbb{N}_{>0}$. The set of all such elements is the integral closure $\bar{S}_{\Lambda}$ of $S$ in $\Lambda$. For a monoid $S$, consider the group $\mathbb{Z} S$, which is the smallest group containing $S$. Then $S$ is called normal if it equals its own normalisation $\bar{S}:=\bar{S}_{\mathbb{Z} S}$, i.e. $S=\bar{S}$. Let us consider the following two examples:

Example 1 (non-normal). Let $S_{1} \subset \mathbb{Z}^{2}$ be the monoid generated by $\{(4,0),(3,1),(1,3),(0,4)\}$, see figure 5 . We observe that the point $(2,2)$ does not lie in $S_{1}$, but in $\mathbb{Z} S_{1}$. This follows from various forms

$$
(2,2)=2 \cdot(3,1)-(4,0) \quad \text { or } \quad(2,2)=2 \cdot(1,3)-(0,4) .
$$

By definition of normality, we see that $2 \cdot(2,2) \in S_{1}$ while $(2,2) \notin S_{1}$, implying that $S_{1}$ is not normal. Therefore, $\mathbb{K}\left[S_{1}\right]$ is neither normal nor Cohen-Macaulay. The geometric reason behind is that $S_{1}$ does not originate from a strongly convex polyhedral cone. Nevertheless, we can provide the explicit description of the monoid ring as follows

$$
\begin{aligned}
& R_{1} \equiv \mathbb{K}\left[S_{1}\right] \cong \mathbb{K}\left[z_{1}, z_{2}, z_{3}, z_{4}\right] /\left\langle z_{2} z_{3}-z_{1} z_{4}, z_{3}^{3}-z_{2} z_{4}^{2}, z_{1} z_{3}^{2}-z_{2}^{2} z_{4}, z_{2}^{3}-z_{1}^{2} z_{3}\right\rangle, \\
& \text { for } \quad z_{1}=x^{(4,0)}, z_{2}=x^{(3,1)}, z_{3}=x^{(1,3)}, z_{4}=x^{(0,4)} .
\end{aligned}
$$

As a remark, such a non-normal ring would have a peculiar appearance in physics, as there exists a certain operator which is not in the chiral ring, but any power of it is an element of the chiral ring. Nevertheless, physical realisations of non-normal varieties appeared, for example, as Higgs branches in [38], based on the mathematical results of [39].

Example 2 (normal and Cohen-Macaulay). Let $S_{2} \subset \mathbb{Z}^{2}$ be the the 2-dimensional monoid generated by $\{(1,2),(1,1),(1,0),(1,-1)\}$, see figure 6 . It is apparent that $S_{2}$ is the monoid 


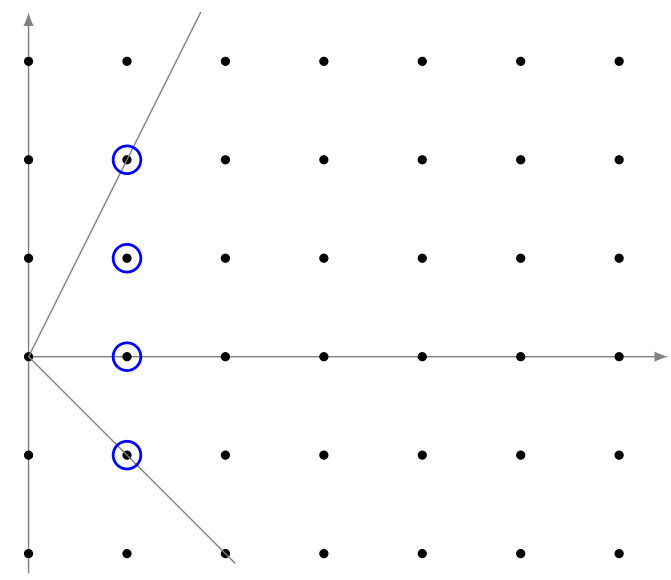

Figure 6. The monoid $S_{2}$ spanned by $\{(1,2),(1,1),(1,0),(1,-1)\}$ and the blue circled points are the elements of the Hilbert basis.

associated to the cone Cone $\{(1,2),(1,-1)\}$ by intersection with the lattice $\mathbb{Z}^{2}$. Since the cone is strongly convex, $S_{2}$ is normal. Therefore, $\mathbb{K}\left[S_{2}\right]$ is normal as well as CohenMacaulay. In more detail, the monoid algebra is given by

$$
\begin{gathered}
R_{2} \equiv \mathbb{K}\left[S_{2}\right] \cong \mathbb{K}\left[z_{1}, z_{2}, z_{3}, z_{4}\right] /\left\langle z_{3}^{2}-z_{2} z_{4}, z_{2} z_{3}-z_{1} z_{4}, z_{2}^{2}-z_{1} z_{3}\right\rangle \\
\text { for } \quad z_{1}=x^{(1,2)}, z_{2}=x^{(1,1)}, z_{3}=x^{(1,0)}, z_{4}=x^{(1,-1)} .
\end{gathered}
$$

Of fundamental importance for the study of a Cohen-Macaulay ring $R$ is the canonical module $\omega_{R}$ of it. We refrain from the generic definition [25, Def. 3.3.1, p. 107] and settle on the statements valid for monoid algebras $R=\mathbb{K}[S]$. In general, the existence and uniqueness of $\omega_{R}$ has to be addressed; however, for normal monoid rings the canonical module exists and equals the unique ideal spanned by the monomials $x^{\nu}$ with $\nu \in \operatorname{Relint}(S)$. In the simplest case, we consider the monoid $S$ spanned by $\{(1,0),(0,1)\}$, for which $\mathbb{K}[S] \cong$ $\mathbb{K}\left[z_{1}, z_{2}\right]$ for $z_{1}=x^{(1,0)}$ and $z_{2}=x^{(0,1)}$. Then $\operatorname{Relint}(S)=\left\{\left(\nu_{1}, \nu_{2}\right) \in \mathbb{N}^{2} \mid \nu_{1}>0, \nu_{2}>0\right\}$ such that $\omega_{R}=\left\langle z_{1} \cdot z_{2}\right\rangle$.

An interesting sub-class of Cohen-Macaulay rings are so-called Gorenstein rings. The defining property is that the ring is (isomorphic to) its own canonical module. For a monoid ring this translates into the condition: there exists a $\nu \in \operatorname{Relint}(S)$ such that $\operatorname{Relint}(S)=\nu+S$. Let us consider the following example:

Example 3 (Gorenstein). Let $S_{3} \subset \mathbb{Z}^{2}$ be the monoid spanned by $\{(1,1),(1,0),(1,-1)\}$, as depcited in figure $\%$. It is convenient represent points in the two sub-monoids $S_{3} \cap\left\{ \pm \nu_{2} \geq 0\right\}$ via

$$
\begin{aligned}
& \left(\nu_{1}, \nu_{2}\right)=\left(\nu_{1}-\nu_{2}\right) \cdot(1,0)+\nu_{2} \cdot(1,1) \quad \text { for } \quad \nu_{1} \geq \nu_{2} \geq 0, \\
& \left(\nu_{1},-\nu_{2}\right)=\left(\nu_{1}-\nu_{2}\right) \cdot(1,0)+\nu_{2} \cdot(1,-1) \quad \text { for } \quad \nu_{1} \geq \nu_{2} \geq 0 \text {. }
\end{aligned}
$$

In other words, the monoid $S_{3}$ is sort of foliated along the 1-dimensional monoid spanned by $(1,0)$, which then implies that

$$
\operatorname{Relint}\left(S_{3}\right)=(1,0)+S_{3} .
$$




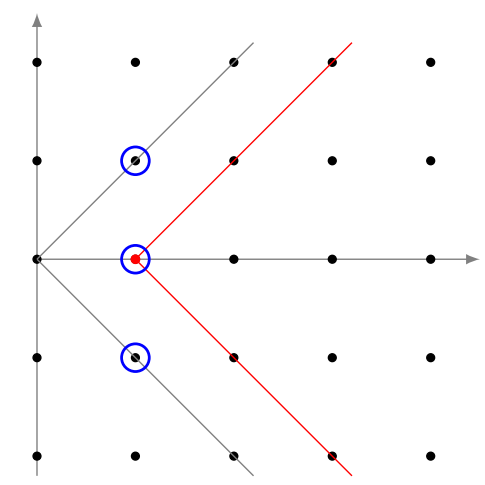

Figure 7. The monoid $S_{3}$ spanned by $\{(1,1),(1,0),(1,-1)\}$ and the blue circled points are the elements of the Hilbert basis. The red point $(1,0)$ is a point in the relative interior such that the shift of $S_{3}$ by $(1,0)$ (denoted by the red lines) equals the relative interior.

Therefore, the monoid algebra $\mathbb{K}\left[S_{3}\right]$ is not only Cohen-Macaulay, but also Gorenstein. In detail

$$
\begin{gathered}
R_{3} \equiv \mathbb{K}\left[S_{3}\right] \cong \mathbb{K}\left[z_{1}, z_{2}, z_{3}\right] /\left\langle z_{1} \cdot z_{2}-z_{3}^{2}\right\rangle \quad \text { and } \quad \omega_{R_{3}}=\left\langle z_{3}\right\rangle \equiv z_{3} \mathbb{K}\left[S_{3}\right] \\
\text { for } \quad z_{1}=x^{(1,1)}, \quad z_{2}=x^{(1,-1)}, \quad z_{3}=x^{(1,0)}
\end{gathered}
$$

Thus, the canonical module is just a translate of $R_{3}$, i.e. all elements are shifted in degree by $(1,0)$.

Open Access. This article is distributed under the terms of the Creative Commons Attribution License (CC-BY 4.0), which permits any use, distribution and reproduction in any medium, provided the original author(s) and source are credited.

\section{References}

[1] S. Cremonesi, A. Hanany and A. Zaffaroni, Monopole operators and Hilbert series of Coulomb branches of $3 d \mathcal{N}=4$ gauge theories, JHEP 01 (2014) 005 [arXiv:1309.2657] [INSPIRE].

[2] S. Cremonesi, A. Hanany, N. Mekareeya and A. Zaffaroni, Coulomb branch Hilbert series and three dimensional Sicilian theories, JHEP 09 (2014) 185 [arXiv:1403.2384] [INSPIRE].

[3] S. Cremonesi, A. Hanany, N. Mekareeya and A. Zaffaroni, Coulomb branch Hilbert series and Hall-Littlewood polynomials, JHEP 09 (2014) 178 [arXiv:1403.0585] [INSPIRE].

[4] S. Cremonesi, G. Ferlito, A. Hanany and N. Mekareeya, Coulomb branch and the moduli space of instantons, JHEP 12 (2014) 103 [arXiv: 1408.6835] [INSPIRE].

[5] S. Cremonesi, A. Hanany, N. Mekareeya and A. Zaffaroni, $T_{\rho}^{\sigma}(G)$ theories and their Hilbert series, JHEP 01 (2015) 150 [arXiv:1410.1548] [INSPIRE].

[6] A. Hanany and M. Sperling, Coulomb branches for rank 2 gauge groups in $3 d \mathcal{N}=4$ gauge theories, JHEP 08 (2016) 016 [arXiv:1605.00010] [INSPIRE].

[7] A. Hanany, C. Hwang, H. Kim, J. Park and R.-K. Seong, Hilbert series for theories with aharony duals, JHEP 11 (2015) 132 [arXiv:1505.02160] [INSPIRE]. 
[8] S. Cremonesi, The Hilbert series of $3 d \mathcal{N}=2$ Yang-Mills theories with vectorlike matter, J. Phys. A 48 (2015) 455401 [arXiv: 1505.02409] [InSPIRE].

[9] S. Cremonesi, N. Mekareeya and A. Zaffaroni, The moduli spaces of $3 d \mathcal{N} \geq 2$ Chern-Simons gauge theories and their Hilbert series, JHEP 10 (2016) 046 [arXiv: 1607. 05728] [INSPIRE].

[10] F. Carta and H. Hayashi, Hilbert series and mixed branches of $T[\mathrm{SU}(N)]$ theory, arXiv: 1609.08034 [INSPIRE].

[11] H. Nakajima, Towards a mathematical definition of Coulomb branches of 3-dimensional $\mathcal{N}=4$ gauge theories, I, Adv. Theor. Math. Phys. 20 (2016) 595 [arXiv:1503.03676] [INSPIRE].

[12] H. Nakajima, Questions on provisional Coulomb branches of 3-dimensional $\mathcal{N}=4$ gauge theories, arXiv: 1510.03908 [INSPIRE].

[13] A. Braverman, M. Finkelberg and H. Nakajima, Towards a mathematical definition of Coulomb branches of 3 -dimensional $\mathcal{N}=4$ gauge theories, II, arXiv:1601.03586 [INSPIRE].

[14] M. Bullimore, T. Dimofte and D. Gaiotto, The Coulomb branch of $3 d \mathcal{N}=4$ theories, arXiv: 1503.04817 [INSPIRE].

[15] M. Bullimore, T. Dimofte, D. Gaiotto, J. Hilburn and H.-C. Kim, Vortices and Vermas, arXiv: 1609.04406 [INSPIRE].

[16] P. Goddard, J. Nuyts and D.I. Olive, Gauge theories and magnetic charge, Nucl. Phys. B 125 (1977) 1 [INSPIRE].

[17] F. Englert and P. Windey, Quantization condition for 't Hooft monopoles in compact simple Lie groups, Phys. Rev. D 14 (1976) 2728 [INSPIRE].

[18] G. 't Hooft, On the phase transition towards permanent quark confinement, Nucl. Phys. B 138 (1978) 1 [INSPIRE].

[19] V. Borokhov, A. Kapustin and X.-k. Wu, Monopole operators and mirror symmetry in three-dimensions, JHEP 12 (2002) 044 [hep-th/0207074] [INSPIRE].

[20] V. Borokhov, A. Kapustin and X.-k. Wu, Topological disorder operators in three-dimensional conformal field theory, JHEP 11 (2002) 049 [hep-th/0206054] [INSPIRE].

[21] D. Gaiotto and E. Witten, S-duality of boundary conditions in $N=4$ super Yang-Mills theory, Adv. Theor. Math. Phys. 13 (2009) 721 [arXiv:0807.3720] [InSPIRE].

[22] M.K. Benna, I.R. Klebanov and T. Klose, Charges of monopole operators in Chern-Simons Yang-Mills theory, JHEP 01 (2010) 110 [arXiv:0906.3008] [INSPIRE].

[23] D. Bashkirov and A. Kapustin, Supersymmetry enhancement by monopole operators, JHEP 05 (2011) 015 [arXiv: 1007.4861] [INSPIRE].

[24] G.M. Ziegler, Lectures on polytopes, updated $7^{\text {th }}$ printing of the $1^{\text {st }}$ edition, Graduate Texts in Mathematics volume 152, Springer, Germany (1995).

[25] W. Bruns and H.J. Herzog, Cohen-Macaulay rings, Cambridge University Press, Cambridge U.K. (1998).

[26] R. Koch, Affine monoids, Hilbert bases and Hilbert functions, Ph.D. thesis, Universität Osnabrück, Osnabrück, Germany (2003).

[27] W. Bruns and J. Gubeladze, Polytopes, rings, and K-theory, Springer Monographs in Mathematics, Springer, Germany (2009). 
[28] E. Miller and B. Sturmfels, Combinatorial commutative algebra, Graduate Texts in Mathematics volume 227, Springer, Germany (2005).

[29] A. Sebö, Hilbert bases, caratheodory's theorem and combinatorial optimization, in the proceedings of the $1^{\text {st }}$ Integer Programming and Combinatorial Optimization Conference, May 28-30, Ontario, Canada (1990)

[30] D. Cox, J. Little, and D. O'Shea, Using algebraic geometry, Graduate Texts in Mathematics volume 227, Springer, Germany (1998).

[31] R.P. Stanley, Hilbert functions of graded algebras, Adv. Math. 28 (1978) 57.

[32] G. Ferlito and A. Hanany, A tale of two cones: the Higgs branch of $\operatorname{Sp}(n)$ theories with $2 n$ flavours, arXiv:1609.06724 [INSPIRE].

[33] D. Eisenbud, D.R. Grayson, M.E. Stillman and B. Sturmfels, Computations in algebraic geometry with Macaulay 2, Algorithms and Computations in Mathematics volume 8, Springer, Germany (2001).

[34] R. Birkner, Polyhedra: a package for computations with convex polyhedral objects, J. Softw. Alg. Geom. 1 (2009) 11.

[35] S. Benvenuti, A. Hanany and N. Mekareeya, The Hilbert series of the one instanton moduli space, JHEP 06 (2010) 100 [arXiv:1005.3026] [INSPIRE].

[36] S. Cabrera and A. Hanany, Branes and the Kraft-Procesi transition, JHEP 11 (2016) 175 [arXiv: 1609.07798] [INSPIRE].

[37] D. Cox, J. Little and H. Schenck, Toric varieties, Graduate Studies in Mathematics volume 124, American Mathematical Society, U.S.A. (2011).

[38] A. Hanany and R. Kalveks, Quiver theories for moduli spaces of classical group nilpotent orbits, JHEP 06 (2016) 130 [arXiv:1601.04020] [INSPIRE].

[39] H. Kraft and C. Procesi, On the geometry of conjugacy classes in classical groups, Comment. Math. Helv. 57 (1982) 539. 